hep-ph/0101106

MADPH-00-1207

TPI-00164

UPR-916-T

\title{
CP-Violating Phases in SUSY, Electric Dipole Moments, and Linear Colliders
}

\author{
V. Barger, T. Falk,凹 T. Han, J. Jiang, \\ T. Li, [円 and T. Plehn \\ Department of Physics, University of Wisconsin, Madison, WI 53706, USA
}

\begin{abstract}
We reexamine large CP-violating phases in the general Minimal Supersymmetric Standard Model, as well as more restricted models. We perform a detailed scan over parameter space to find solutions which satisfy the current experimental limits on the electric dipole moments of the electron, neutron and ${ }^{199} \mathrm{Hg}$ atom, exploring the allowed configurations of phases and masses, and we attempt to quantify the level of tuning of the parameters necessary to populate the regions of cancellations. We then consider the measurement of CP-violating phases at a future linear collider. We find that measurements of chargino and neutralino masses and production crosssections allow for a determination of $\phi_{1}$ (the phase of $M_{1}$ ) to a precision of $\pi / 30$, while the EDM constraints require that $\theta_{\mu}$ be too small to be measured. Using the EDM constraints we find that the CP-even model parameters and the phase $\phi_{1}$ can be determined at a Linear Collider with $400 \mathrm{GeV}$ c.m. energy. As long as some information on the size of $|\mu|$ is included in the observables, a measurement of $\phi_{1}$ is guaranteed for $\phi_{1}>\pi / 10$. To unambiguously identify CP violation, we construct CP-odd kinematical variables at a linear collider. However, the CP asymmetries are rather small, typically about $0.1-1.5 \%$, and it will be challenging to experimentally observe the predicted asymmetries.

\footnotetext{
${ }^{1}$ Current address: Theoretical Physics Institute, School of Physics and Astronomy, University of Minnesota, Minneapolis, MN 55455

${ }^{2}$ Current address: Department of Physics and Astronomy, University of Pennsylvania, Philadelphia, PA 19104
} 


\section{Introduction}

In the last two years, there has been a resurgence of interest in CP-violating phases in supersymmetric theories. Although in many cases the size of the CP-violating phases is strongly constrained by limits on the electric dipole moments (EDMs) of the electron, neutron and ${ }^{199} \mathrm{Hg}$ atom, possible cancellations between different contributions to the EDMs can significantly weaken the upper bounds on the phases [1], even potentially allowing phases of $\mathcal{O}(1)$ [2, 3], typically in small, often finely tuned regions. This observation has led to an explosion of papers exploring the consequences of the presence of large phases, many papers finding some regions of parameter space where large phases are of consequence to the system under study, but few papers simultaneously imposing the (still severe) constraints from EDMs. One of the most interesting questions is whether one will be able to measure the phases in chargino and neutralino production at a future linear collider 4, 5]. Even if one expects that weakly interacting supersymmetric partners are going to be found at the Tevatron and at the LHC [6, 7], the model parameters have to be determined in detail in a precision experiment. A future high luminosity Linear Collider is the optimal tool to extend the LEP Standard Model precision measurements to other models. The CP-conserving set of underlying MSSM parameters can be easily determined from physical masses alone, cross sections and asymmetries [8]. The effects of CP-violating phases on neutralino and chargino observables can be considerably smaller and therefore require a more ambitious collider energy and luminosity [由, 5].

In this paper we reexamine this question, taking into account the limits from EDM experiments, including the recent improved limits on the ${ }^{199} \mathrm{Hg}$ EDM [9]. We perform a detailed scan of parameter space in the general (non-unified) MSSM, including phases and masses, to find the regions satisfying the EDM constraints. Using these constraints we show how the phase $\phi_{1}$ can be extracted from a set of CP-even variables (masses and total cross sections) at a future Linear Collider. Compared to the previous analysis [4] we can reduce the requirements on the energy as well as on the luminosity of the Linear Collider. Moreover, we show how one could use an extended set of parameters, which allows for an independent extraction of $|\mu|$, and further relax the requirement on the Collider design parameters. Finally, we construct CP-odd variables at $e^{+} e^{-}$linear colliders. We find that the CP asymmetry is typically about $0.1-1.5 \%$.

\section{CP Violation and Electric Dipole Moments}

The Minimal Supersymmetric Standard Model (MSSM) contains several sources of CP violation not present in the Standard Model. In the most general flavor non-preserving SUSY model, there are over 40 new complex phases [10], although most of the new parameters are very strongly constrained by limits on flavor violating processes. We assume a Peccei-Quinn symmetry [11] 
which sets the coefficient $\bar{\theta}$ of the $\tilde{G} G$ term to zero, up to small corrections coming from higher dimensional CP-violating operators, which shift the minimum of the axion potential [12]. In the MSSM, new complex phases arise in the Higgs mixing mass $\mu$, as well as in the soft SUSY-breaking terms in the Lagrangian: the trilinear scalar mixing masses $A_{i}$, the bilinear Higgs mixing parameter $B$, and the three gaugino mass parameters $M_{i}$. Not all of these phases are physical, however, and some or most may be removed by field redefinitions, depending on the model. In mSUGRA, for example, the phases $\phi_{i}$ of all the $M_{i}$ may be removed by an R-rotation' at the unification scale $M_{X}$, the $A_{i}$ are united to a common $A_{0}$ at $M_{X}$, and a rotation of one of the Higgs fields can be used to set $\theta_{\mu}+\theta_{B}=0$, so that the Higgs vevs are real. This leaves only two physical phases in mSUGRA, which can be taken to be $\theta_{A_{0}}$ and $\theta_{\mu}$.

In more general models, where the $M_{i}$ do not unify, only one of the gaugino phases can be rotated away, which we take to be the phase of $M_{2}$. In our more general analyses, we will also take independent phases in the trilinear parameters $A_{e}, A_{u}, A_{d}$ and $A_{t}$. This leaves us with seven physical phases in our most general model. We emphasize that these are the phases present after phases have been removed in the field redefinitions described above. Therefore when we constrain below the phase of the $\mu$ parameter to be very small, it is in fact some combination of the phases in the original parameterization which is restricted. This is particularly important in models where there is a correlation between the phase of $B$ and the other phases in the model, such as in mSUGRA, as we discuss further below.

It is well known that the additional sources of CP violation in supersymmetric models can contribute to the electric dipole moments of the neutron and electron [13, 14] and mercury atom [15]. The very tight experimental limits on these quantities [16, 17, 9]

$$
\begin{aligned}
d_{n} & <1.1 \times 10^{-25} \mathrm{e} \cdot \mathrm{cm} \\
d_{e} & <4 \times 10^{-27} \mathrm{e} \cdot \mathrm{cm} \\
d_{\mathrm{Hg}} & <2 \times 10^{-28} \mathrm{e} \cdot \mathrm{cm}
\end{aligned}
$$

then impose severe constraints on the CP-violating phases in SUSY models. To suppress the EDMs, either large scalar masses $\left(>1 \mathrm{TeV}\right.$ ) or small phases (of the order $10^{-3}$, when all SUSY masses are of order $100 \mathrm{GeV}$ ) are typically required. However, as pointed out in [19, 1, 18], such large scalar masses are cosmologically problematic, and the addition of cosmological constraints to the mix implies that (some of) the CP-violating phases are constrained to be quite small when the LSP is a dominantly $\widetilde{B}$-type neutralino.

The electron EDM receives contributions from chargino and neutralino exchange diagrams, shown in Fig. 1. The full expressions for the dependence of the induced electron EDM on the

\footnotetext{
${ }^{1}$ Under an R-rotation of the fields, the $A_{i}, B$ and $M_{i}$ effectively pick up a common phase, while the other soft SUSY breaking masses and $\mu$ are left unchanged.
} 
SUSY masses and phases can be found in [14, 2]. The dominant contribution is typically from the chargino exchange diagram and is proportional to $\sin \theta_{\mu}$. Thus the primary constraint coming from the electron EDM limits is an upper limit on the phase of $\mu$. The subdominant neutralino

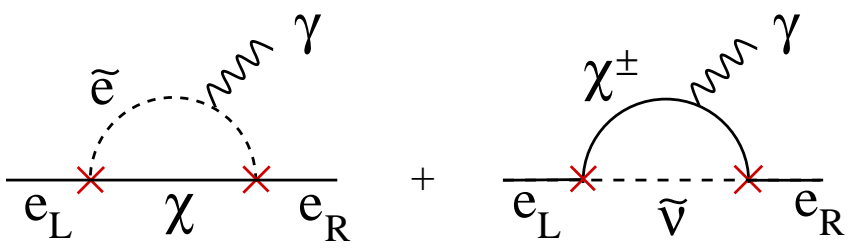

Figure 1: Diagrams contributing to the electric dipole moment of the electron. The SUSY phases enter at the crossed vertices.

exchange piece has a more complicated dependence on the SUSY phases, including both pieces proportional to $\sin \theta_{\mu}$ and $\sin \gamma_{e}$, where $\gamma_{e}=\arg \left(A_{e}+\mu^{*} \tan \beta\right)$. Cancellations can occur between the neutralino chargino exchange contributions, and this serves to weaken the absolute limits on the SUSY phases, although $\theta_{\mu}$ of $\mathcal{O}(1)$ requires either severe fine-tuning of parameters or a very heavy spectrum, as we will see below.

The neutron EDM is considerably more complicated, and until recently, the computation 14, 2] of the neutron EDM induced by SUSY phases has been plagued by very large theoretical uncertainties. The SUSY phases contribute both to the EDMs and color EDMs (cEDMs) of the quarks, and in the last year, the contribution to the neutron EDM both from the induced $\bar{\theta}$ due to the color EDMs of the quarks [20] and from the quark EDMs and cEDMs themselves [21] have been reliably calculated using QCD sum rules, allowing a reduction in the theoretical uncertainty. The overall effect is to reduce slightly the predicted neutron EDM, and with smaller error bars. The quark EDMs receive contributions from the chargino and neutralino exchange diagrams of

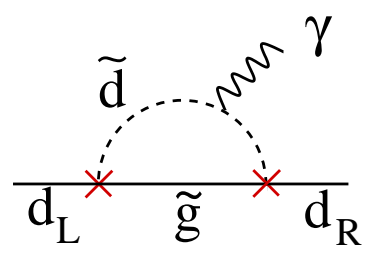

Figure 2: Diagram contributing to the electric dipole moment of the down quark. The SUSY phases enter at the crossed vertices.

Fig. 1 (where the photon can now also connect to the sfermion line in the chargino diagram), as well as from the gluino exchange diagram of Fig. \&. The gluino exchange contribution to the 
$d$-quark EDM is proportional to $\gamma_{d}=\arg \left(A_{d}+\mu^{*} \tan \beta\right.$ ) (for the $u$-quark take $\tan \beta \rightarrow \cot \beta$ ). There are also significant contributions to the neutron EDM from the color dipole moments of the quarks, which have the same dependence on the phases at the quark EDMs, and are of the same order as the contribution from the induced $\bar{\theta}$ term described above. The neutron EDM written in terms of the quark EDMs and cEDMs (which can be found in [2, 14]) is given in [21]. Lastly, there is also a small contribution from the three-gluon operator $O_{G}=-\frac{1}{6} f^{a b c} G_{a} G_{b} \tilde{G}_{c}$ [22]. Two-loop contributions to the EDMs may be significant at very large values of $\tan \beta$ [23]. For the values of $\tan \beta$ we consider, the effect of higher order contributions will be to very slightly shift the allowed regions of parameter space.

For the ${ }^{199} \mathrm{Hg}$ atom, the electric screening of the electric dipole moments of the atom's constituents is violated by the finite size of the nucleus and can be conveniently expressed by the Schiff moment $S$, which parameterizes the effective CP-odd interaction between the electron and nucleus of spin $I$. The Schiff moment, in turn, can be induced either through the Schiff moment of the valence nucleons or through the breaking of time reversal invariance in the nucleon-nucleon interaction, the latter being enhanced by the collective effects in the nucleus, and hence is dominant. The largest contributions to the Schiff moment, and hence to the EDM of mercury, are through the color EDMs of the quarks, and the expression for $d_{\mathrm{Hg}}$ in terms of the quark cEDMs can be found in [15]. We emphasize that the mercury, electron and neutron EDMs all depend on different combinations of phases, and so simultaneously imposing all three EDM constraints excludes a much greater portion of the CP-violating SUSY parameter space than from imposing any single constraint.

Again, the EDMs generated by the SUSY phases are sufficiently small if either 1) the phases are very small $\left(\lesssim 10^{-2}-10^{-3}\right)$, or 2$)$ the SUSY masses are very large $(\mathcal{O}($ a few $\mathrm{TeV}))$, or 3$)$ there are large cancellations between different contributions to the EDMs, or by a combination of these effects. It is condition 3), large cancellations between different contributions to the electric dipole moments, which has spurred the greatest interest recently, since it ostensibly allows for both large amounts of CP violation and a spectrum which is phenomenologically relevant. Such cancellations are not easy to achieve, however. If the phases are $\mathcal{O}(1)$ and the SUSY masses are in the $100 \mathrm{GeV}$ range, then the EDM limits will be violated by several orders of magnitude unless very delicate cancellations between the various contributions exist. Further, the parameters must be tuned so that similarly delicate cancellations occur for electron and neutron and $\mathrm{Hg}$ EDMs.

In some models it is impossible to achieve sufficient cancellations in all three EDMs to permit some phases to be $\mathcal{O}(1)$, but even so, the effect of cancellations may still be significant. For example, cancellations do occur naturally in mSUGRA models over significant regions of parameter space [1, 2, 18, 15], including in the body of the cosmologically allowed region with $m_{1 / 2}=$ $\mathcal{O}(100-400 \mathrm{GeV})$. With a $\widetilde{B}$-type neutralino LSP, large sparticle masses cannot be invoked to 
permit large phases, due to limits on the LSP relic abundance [19, 1, 18], and so the phases, in particular $\theta_{\mu}$, are severely limited by the EDMs. The presence of cancellations relaxes the constraints on the phases, but the limit on $\theta_{\mu}$ remains small, $\theta_{\mu} \lesssim \pi / 10$, unless the sfermions (including selectrons) are heavier than $\mathcal{O}(1 \mathrm{TeV})$. This upper bound on $\theta_{\mu}$ is over an order of magnitude less restrictive than what would find in the absence of any cancellations, however, and thus the effect of cancellations is quite significant. We will discuss the role of cancellations in the MSSM in more detail in Section 3.2 .

Minimal SUGRA is a particularly restrictive model in that there are only two new physical phases. In more general models there are more phases, and studies have been made to examine the new cancellations which the presence of additional phases allow, particularly in models without gaugino mass unification, where the gaugino masses $M_{i}$ can have independent phases (see e.g. [3, 24]). The possibility of having both $\mathcal{O}(1)$ phases and reasonable sparticle masses in these models has inspired a remarkable number of recent papers exploring the consequences (phenomenological and cosmological) of new large sources of CP violation in SUSY models. Most of these analyses take the possibility of cancellations as carte blanche to consider all sets of masses and phases, without actually imposing the rigorous constraints on the SUSY parameters from the electric dipole moments. In the next section we will examine the size of the phases one may reasonably expect to satisfy the EDM constraints, and for what sparticle masses, in both mSUGRA and in two more general models without gaugino mass unification. In particular, we will study the level of tuning required to obtain large phases.

\section{EDM Analysis}

We begin by studying the constraints imposed by the EDMs in three different models: one mSUGRA-inspired model with two physical phases, and two models without gaugino mass unification, which have seven independent phases each and 15(23) total free parameters, including masses. We have done large Monte Carlo studies for each case, evaluating roughly 800,000 parameter sets in mSUGRA and 300 and 600 million sets in each of the two more general models respectively, and studied the configurations which satisfy the experimental limits on the EDMs. Table 1 displays the number of parameter sets studied and total number of points satisfying the EDM constraints (11)-(3).

\section{1 mSUGRA-inspired Model}

After performing the field redefinitions described in Section 2, the mSUGRA-like model is specified by 6 parameters: three masses $\left(m_{0}, m_{1 / 2}\right.$, and $\left.A_{0}\right)$, two phases $\left(\theta_{\mu}\left(M_{X}\right)\right.$ and $\left.\theta_{A}\left(M_{X}\right)\right)$, and $\tan \beta$. 


\begin{tabular}{|l||c|c|c|}
\hline Model & Points run & Points satisfying EDMs & fraction satisfying EDMs \\
\hline \hline mSUGRA & $8 \times 10^{5}$ & 10,000 & $1.2 \times 10^{-2}$ \\
15 parameter MSSM & $3 \times 10^{8}$ & 23,650 & $7.1 \times 10^{-5}$ \\
23 parameter MSSM & $6 \times 10^{8}$ & 13,700 & $2.1 \times 10^{-5}$ \\
\hline
\end{tabular}

Table 1: Monte Carlo studies of electric dipole moments in three models.

Throughout we take $m_{\text {top }}=175 \mathrm{GeV}$. In mSUGRA, once the gaugino, soft scalar masses, $A$ and $B$ terms and phases are given at $M_{X}$, they can be evolved using the renormalization group equations (RGE) to the electroweak scale. As in common practice, we use the one-loop RGEs for the masses and two-loop RGEs for the gauge and Yukawa couplings [25]. The structure of the equations for the gauge couplings, gaugino masses and the diagonal elements of the sfermion masses are such that they are entirely real. The evolutions of the $A_{i}$, however, are more complicated, as the $A_{i}$ pick up both real and imaginary contributions. For example, the evolution of $A_{t}$ is given by

$$
\frac{d A_{t}}{d t}=\frac{1}{8 \pi^{2}}\left(-\frac{16}{3} g_{3}^{2} M_{3}-3 g_{2}^{2} M_{2}-\frac{13}{9} g_{1}^{2} M_{1}+h_{b}^{2} A_{b}+6 h_{t}^{2} A_{t}\right)
$$

Thus, $A_{t}$ receives real contributions proportional to the gaugino masses $M_{i}$ and complex contributions from the heavy generation $A_{i}$, multiplied with the respective Yukawa coupling $h_{i}$. Since the coefficients of the $M_{i}$ are flavor dependent and the coefficients of the $h_{f}^{2} A_{f}$ terms are generation dependent, the phases (and magnitudes) of the $A_{i}$ must therefore be run separately. At one loop, the evolution equation for $\mu$ is given by

$$
\frac{d \mu}{d t}=\frac{\mu}{16 \pi^{2}}\left(-3 g_{2}^{2}-g_{1}^{2}+h_{\tau}^{2}+3 h_{b}^{2}+3 h_{t}^{2}\right)
$$

and the phase of $\mu$ does not run. Finally, the $B$ parameter evolves as

$$
\frac{d B}{d t}=\frac{1}{8 \pi^{2}}\left(-3 g_{2}^{2} M_{2}-g_{1}^{2} M_{1}+h_{\tau}^{2} A_{\tau}+3 h_{b}^{2} A_{b}+3 h_{t}^{2} A_{t}\right) .
$$

After evolving the parameters to the weak scale, the phase of Higgs superfield $H_{2}$ (which gives mass to up-type fermions) can be rotated in such a way as to ensure real expectation values for the Higgs scalars. The rotation changes the phase of $H_{2}$ by an amount $-\left(\theta_{\mu}+\theta_{B}\right)$. Not only is the phase of $\mu$ now fixed at $\theta_{\mu}=-\theta_{B}$, but also the initial phase of $\mu$ is physically irrelevant as it is canceled by the rotation. As emphasized in [26, 27], a large phase in $A$ will induce a phase in $B$ (Eq. (6)), and hence in $\mu$, after the vevs are made real. Therefore even if $\mu$ and $B$ are both real at $M_{X}$, if $\theta_{A}$ is large, the value of $\theta_{\mu}\left(M_{Z}\right)$ contributing to the EDMs may be large (of course this is completely equivalent to keeping the Higgs vevs complex and $\mu$ real). Since $B$ is a free parameter, which is typically determined by the conditions of correct electroweak symmetry 
breaking, $B\left(M_{X}\right)$ can be chosen so that $B \mu\left(M_{Z}\right)$ is nearly real, yielding a small $\theta_{\mu}\left(M_{Z}\right)$ after the Higgs rotation; however, this can involve a significant fine-tuning if $\theta_{A}$ is large [27. In practice, this tuning is typically not worse than at the level of $10 \%$ [18], but it must be emphasized that this is a tuning over-and-above the tuning discussed below. Alternatively, if $\theta_{B}\left(M_{X}\right)$ is taken real [26] and $\theta_{A}$ is large, or if $\theta_{B}\left(M_{X}\right)$ is arbitrary, then the additional fine tuning lies in adjusting the original $\theta_{\mu}^{0}$ close to $-\theta_{B}\left(M_{Z}\right)$.

To study the phases permitted by the current experimental limits (11)-(3) on the neutron, electron and ${ }^{199} \mathrm{Hg}$ EDMs, we perform a Monte Carlo studies, sampling the 6 model parameters in the following ranges:

$\begin{aligned} 0 & \leq \theta_{\mu}, \theta_{A} \leq 2 \pi \\ 50 \mathrm{GeV} & \leq m_{1 / 2} \leq 1 \mathrm{TeV} \\ 0 \mathrm{GeV} & \leq A_{0} \leq 1 \mathrm{TeV} \\ 0 \mathrm{GeV} & \leq m_{0} \leq 1 \mathrm{TeV} \\ 2 & \leq \tan \beta \leq 10\end{aligned}$

and keeping parameter sets satisfying (11)-(3). Limits from particle searches have not been imposed. However, the lower limit on $m_{1 / 2}$ was chosen to remove most of the area excluded by the current LEP chargino mass limit of $103 \mathrm{GeV} 28$. Most of the solutions with $\tan \beta \lesssim 3$ are excluded by the Higgs searches at LEP 28]. Cosmological limits on the relic abundance of the LSP neutralino, which tend to exclude regions with $m_{0} \gtrsim 200 \mathrm{GeV}$, have also not been included, we will comment on these regions later.

The results for our Monte Carlo scan of 800,000 mSUGRA parameter sets are summarized in Fig. 3, where we display the 10,000 sets² satisfying the three EDM constraints (11)-(3). Fig. 3a shows the allowed configurations of $\left\{\theta_{\mu}, \theta_{A}\right\}$. The first obvious point to note is that the range of $\theta_{\mu}$ is severely limited, with no events in this sample having $\left|\theta_{\mu}\right|>0.1 \pi(\bmod \pi)$, while the phase $\theta_{A}$ can take any value. This is due to the fact that the chargino contribution to the electron EDM is typically dominant and depends only on $\theta_{\mu}$; therefore for large $\theta_{\mu}$, the neutralino exchange piece cannot provide sufficient cancellation, regardless of the values of $\theta_{A}$. In Figs. 3 $3 \mathrm{~b}-3 \mathrm{j} \mathrm{d}$, we display the value of $\theta_{\mu}$ versus $m_{1 / 2}, m_{0}$ and $\tan \beta$, respectively. We see that there are many more solutions for large $m_{1 / 2}>500 \mathrm{GeV}$, where the heavy spectrum reduces the individual contributions to the EDMs. We note, however, that almost all such parameter sets lead to a neutralino relic abundance $\Omega_{\tilde{\chi}} h^{2}>0.3$ [15], implying a universe younger than 12 billion years, in contradiction to observational evidence. In Fig. $3 \mathrm{~d}$, we see that most of the large $\theta_{\mu}$ solutions occur also for $\tan \beta \lesssim 3$, which in mSUGRA yield a Higgs scalar too light to be compatible with the negative results from Higgs searches at LEP [28]. Indeed, if we consider only those parameter sets with $m_{1 / 2}<500 \mathrm{GeV}, m_{0}<200 \mathrm{GeV}$ and $\tan \beta>3$, we find no solutions with $\theta_{\mu}>\pi / 20$.

\footnotetext{
${ }^{2}$ To reduce the file size in this and following plots, we do not display points lying underneath covered regions, particularly at low $\theta_{\mu}$. This produces no visible effect on the plots.
} 


\subsection{5-parameter MSSM}

We next consider a more general model which has additional independent phases, and which therefore has greater opportunity for cancellations. We no longer require gaugino mass unification. Therefore, one of the three gaugino masses may still be made real by an R-rotation, but the other two, which we take to be $M_{1}$ and $M_{3}$, may be complex, with phases $\phi_{1}$ and $\phi_{3}$ respectively. We additionally allow independent phases in $A_{d}, A_{u}, A_{e}$ and $A_{t}$, and along with $\theta_{\mu}$, this gives 7 independent phases. The other 8 parameters $\operatorname{are} \tan \beta$, plus the masses: $\left|M_{i}\right|, i=1 \ldots 3$, a common $\left|A_{i}\right|=|A|, i=e, d, u, t,|\mu|$, and the sfermion mass parameters $m_{\tilde{e}_{R}}^{2}, m_{\tilde{u}_{R}}^{2}$. We fix the remaining masses by the approximate relations $m_{\tilde{d}_{R}}^{2}=m_{\tilde{u}_{R}}^{2}, m_{\tilde{e}_{L}}^{2}=m_{\tilde{e}_{R}}^{2}+0.6 M_{2}^{2}$, and $m_{\tilde{q}_{L}}^{2}=m_{\tilde{q}_{R}}^{2}+0.5 M_{2}^{2}$.
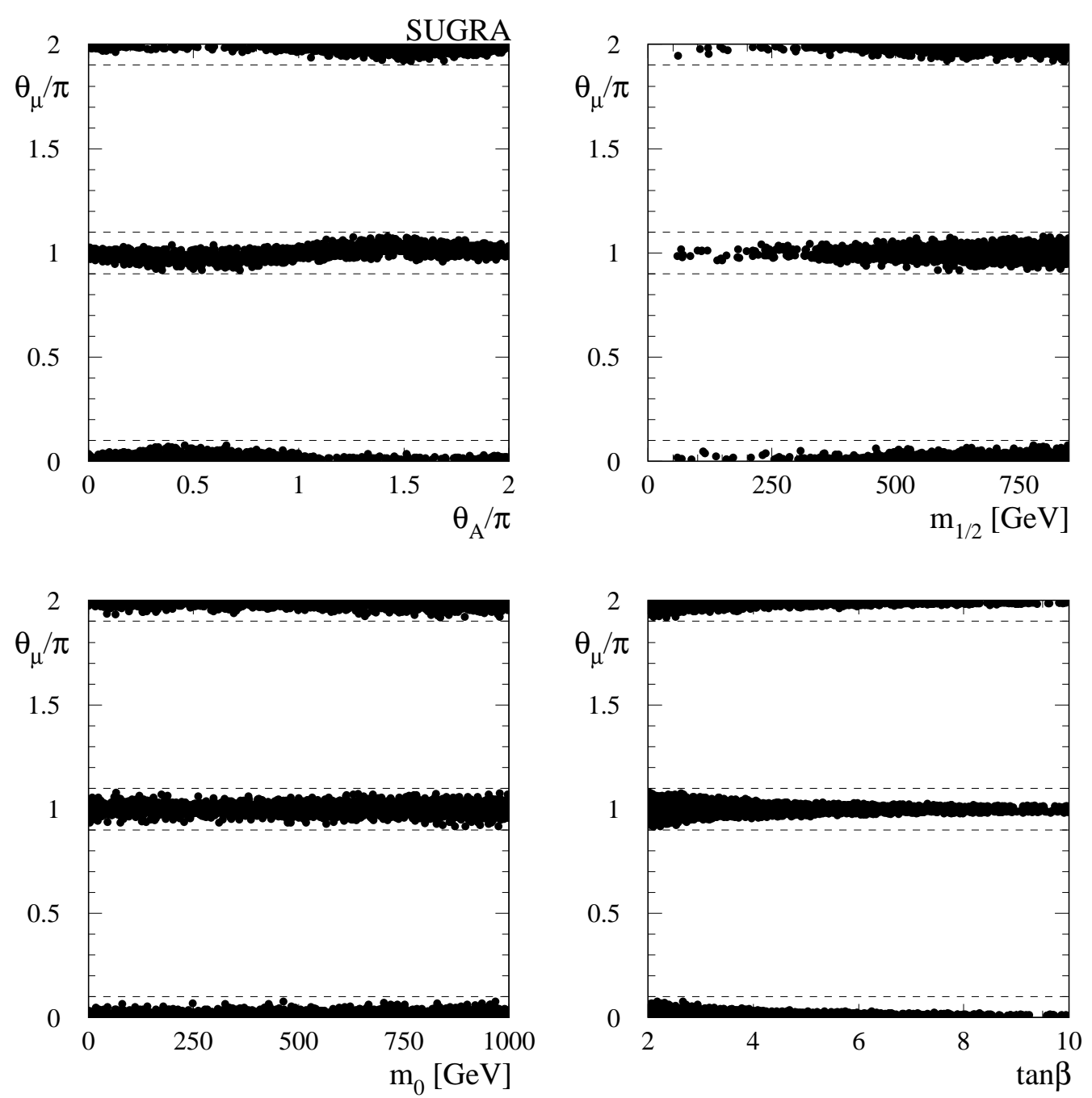

Figure 3: mSUGRA solutions satisfying the experimental limits on the electron, neutron and ${ }^{199} \mathrm{Hg}$ EDMs. 
We then sample the regions

\begin{tabular}{|c|c|c|c|c|}
\hline 0 & $\leq$ & $\theta_{\mu}, \phi_{i}, \theta_{A i}$ & $\leq$ & $2 \pi$ \\
\hline $100 \mathrm{GeV}$ & $\leq$ & $\mu$ & $\leq$ & $1 \mathrm{TeV}$ \\
\hline $100 \mathrm{GeV}$ & $\leq$ & $2 M_{1}, M_{2}, M_{3}$ & $\leq$ & $1 \mathrm{TeV}$ \\
\hline $0 \mathrm{GeV}$ & $\leq$ & $|A|$ & $\leq$ & $1 \mathrm{TeV}$ \\
\hline $\begin{array}{r}0 \mathrm{GeV} \\
2\end{array}$ & $\leq$ & $\begin{array}{c}m_{\tilde{e}_{R}}, m_{\tilde{u}_{R}} \\
\quad \tan \beta\end{array}$ & $\leq$ & $\begin{array}{l}1 \mathrm{TeV} \\
10\end{array}$ \\
\hline
\end{tabular}

and look for parameter sets satisfying (11)-(3). We cut out the (few) remaining sets with $m_{\chi^{ \pm}}<$ $103 \mathrm{GeV}$, and we flag the sets with $m_{h}<113 \mathrm{GeV}$, as discussed below.

Again, because of the additional possibilities for cancellations in this parameterization, we expect solutions with larger values of the phases which still satisfy the EDM constraints (11)-(3). This is borne out in Figs. 46, where we display the results for our Monte Carlo scan of roughly 300 million points in the 15-parameter MSSM. In the top two panels of Fig. 14 we display the 23,500 sets allowed by the EDMs, in the $\left\{\theta_{\mu}, \phi_{1}\right\}$, and $\left\{\theta_{\mu}, \phi_{3}\right\}$ planes, respectively. Although by far the greatest events of solutions have $\theta_{\mu} \lesssim \pi / 10$, there is now a visible swath that extends to large values of $\theta_{\mu}$. The range of $\phi_{1}$ and $\phi_{3}$ are unconstrained, as are the ranges of the $\theta_{A_{i}}$ (not displayed). The presence of the large $\theta_{\mu}$ solutions relies on having large sparticle masses, significant cancellations between contributions to the EDMs, or small $\tan \beta$, and very typically a combination of the above.

In Fig. W we display $\theta_{\mu}$ versus $\tan \beta$ for the parameter sets, and we see clearly the weakened constraint on $\theta_{\mu}$ at low $\tan \beta$. However, most of the low $\tan \beta$ solutions are actually experimentally excluded by the unsuccessful Higgs searches at LEP2. To be conservative, we take the stop soft masses to be independent parameters and set them to $1 \mathrm{TeV}$, take $m_{A}=1 \mathrm{TeV}$, and we compute the light Higgs mass as a function of $\tan \beta$ in the maximal mixing scenario using Ref. [29]. The light (green) points in all four panels of Fig. 四 yield $m_{h}<113 \mathrm{GeV}$ (corresponding to $\tan \beta \lesssim 2.4$ ). Hence, they would require large phases and further tuning in the Higgs sector not to be excluded by LEP [28. In Fig. 5, we display $\theta_{\mu}$ as a function of the light $\left(\tilde{\chi}_{1}^{+}\right)$and heavy $\left(\tilde{\chi}_{2}^{+}\right)$chargino masses. The chargino exchange contribution to the fermion EDMs and cEDMs [14, 2] is suppressed both for highly split charginos and for a heavy chargino spectrum. We see that if neither chargino is heavy $\left(m_{\chi^{ \pm}} \lesssim 500 \mathrm{GeV}\right), \theta_{\mu}$ is strongly constrained, whereas if at least one of the charginos is heavy, $\theta_{\mu}$ can potentially be large. In particular, having a spectrum with only one light chargino does not by itself forbid $\theta_{\mu}>\pi / 10$.

The significance of cancellations on the allowed range of $\theta_{\mu}$ is seen in Fig. 6. In the first panel we display the value of $\theta_{\mu}$ versus the ratio the the chargino $\left(d_{e}^{C}\right)$ and neutralino $\left(d_{e}^{N}\right)$ exchange contributions to the electron EDM. We see the clustering of the large $\theta_{\mu}$ solutions near to the cancellation point $d_{e}^{C} / d_{e}^{N}=-1$. The vertical lines correspond to an electron EDM a factor of 5 

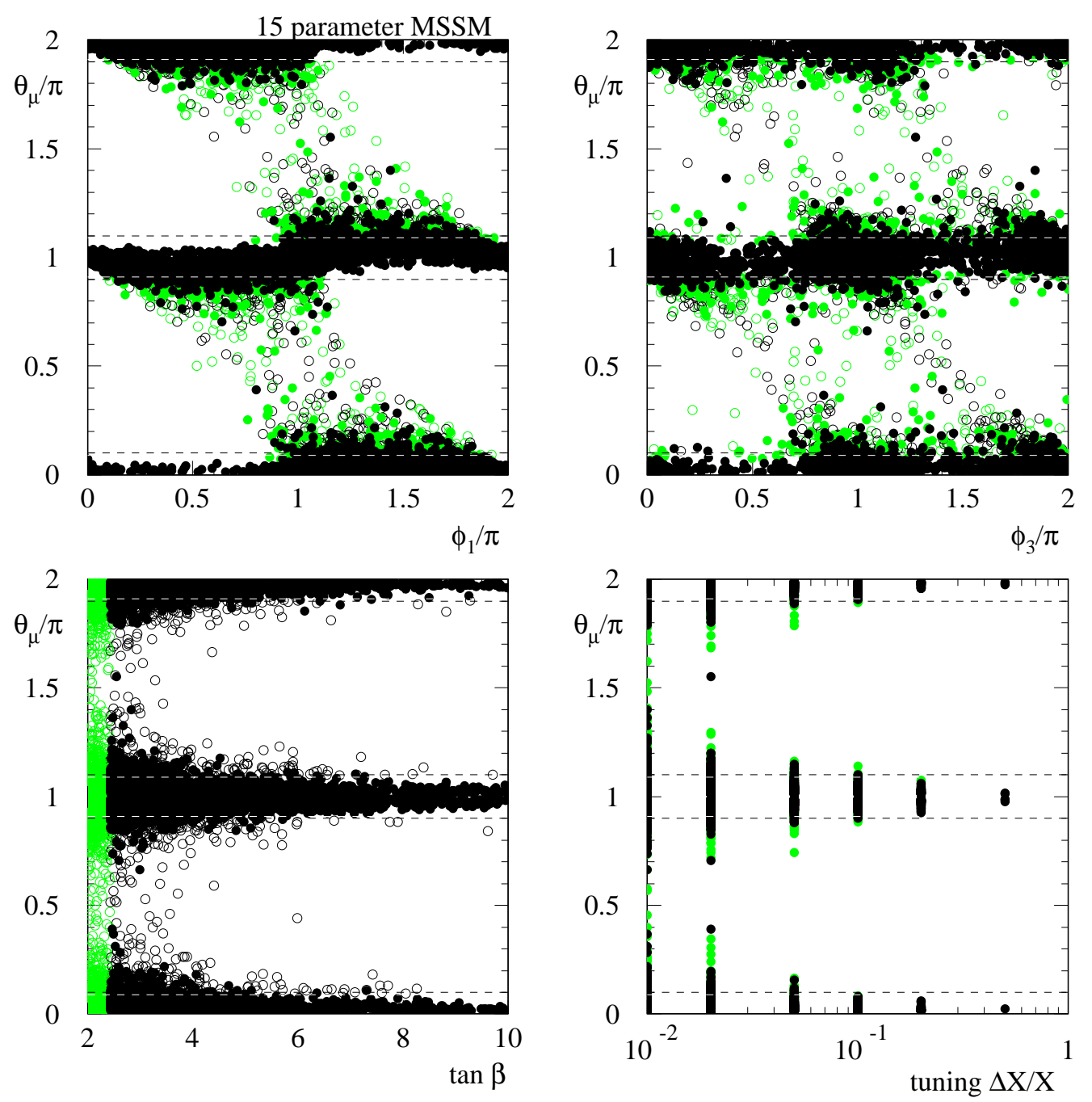

Figure 4: Parameter sets in the 15-parameter MSSM satisfying the experimental limits on the electron, neutron and ${ }^{199} \mathrm{Hg}$ EDMs. Open circles suffer from parameter tuning $\Delta X / X$ worse than 1\% (see the text). Light (green) dots correspond to configurations with a light Higgs $m_{h}<$ $113 \mathrm{GeV}$. The tuning parameter $\Delta X / X$ is defined in the text, and corresponds to the maximum variation that the point survives. 
smaller than the larger of $d_{e}^{C}$ and $d_{e}^{N}$, due to cancellations. This demonstrates that the sparticles must be much heavier than those allowed by our range of parameters $(>1 \mathrm{TeV})$ in order for the EDM constraints to be satisfied for large $\theta_{\mu}$ in the absence of any cancellations. In practice, the large $\theta_{\mu}$ solutions have both some sparticle masses near the upper end of their ranges and a large degree of cancellations. In the second panel of Fig. 6, we display $\theta_{\mu}$ as a function of the sfermion mass $m_{\tilde{e}_{R}}$. Perhaps counter-intuitively, the large $\theta_{\mu}$ solutions tend to lie at lower $m_{\tilde{e}_{R}}$, rather than near the upper end of their range. However, this is again due to the necessity for cancellations: $m_{\tilde{\ell}} \sim 1 \mathrm{TeV}$ is not large enough to sufficiently turn off the SUSY contributions to the electron EDM for large $\theta_{\mu}$, and the necessary cancellations only occur for smaller slepton masses.

One goal of this paper is to study the extent to which the large phase solutions require a significant tuning of the model parameters in order to fall into the regions of EDM cancellations. The tuning measure we employ is simple but intuitive. For every parameter set we find which satisfies the EDM constraints, we perform the following analysis. We begin by varying all the input parameters (one at a time) by $\Delta X / X= \pm 0.5 \%$ and see if the EDM limits are still satisfied for all the test parameter sets. If they are, we then try varying all the input parameters by $\pm 1 \%$, and so on, until we find the smallest percentage change for which the configuration violates one of the EDM bounds. This gives a sense of the local "size" of the allowed parameter region. The results for the 15-parameter MSSM scan are displayed in the final panel of Fig. 4, where we have stepped through $0.5 \%, 1 \%, 2 \%, 5 \%, 10 \%, 20 \%$ and $50 \%$ changes, modulo $\pi$ in the case of phases, and the last percentage change to the underlying parameters that successfully satisfies the EDM
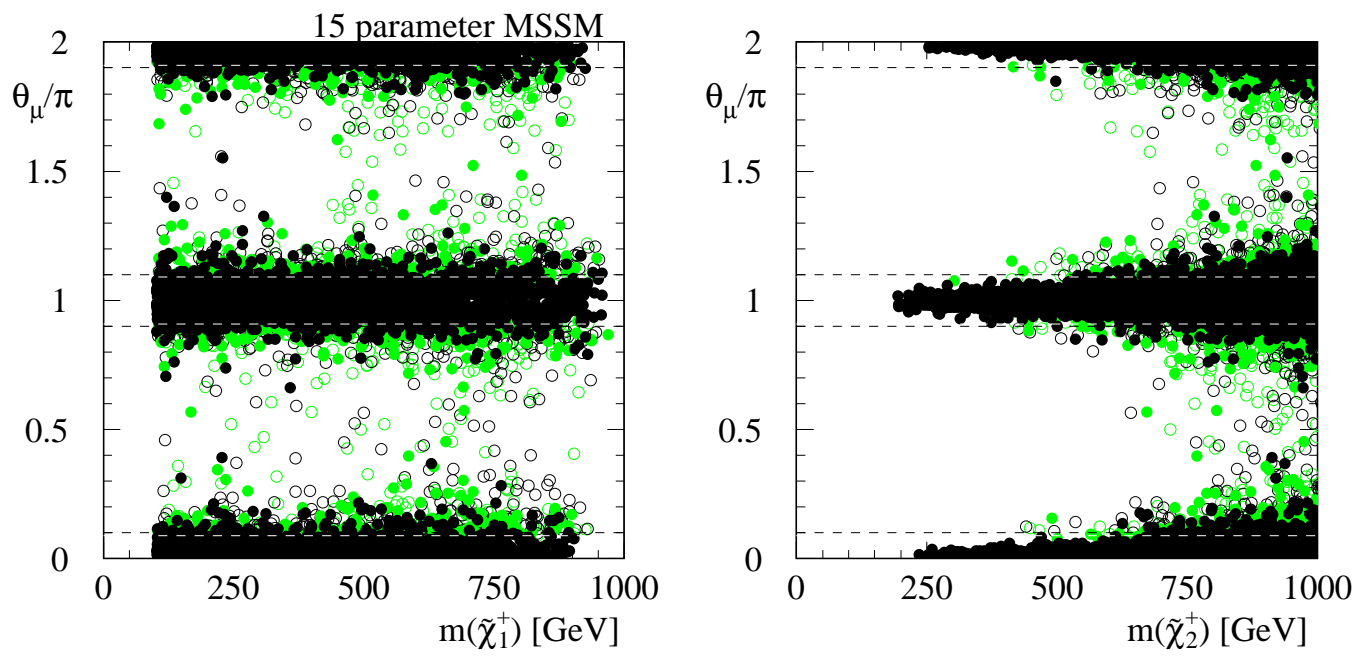

Figure 5: For the 15-parameter MSSM scan, the dependence of the solutions on the light and heavy chargino masses. Open circles suffer from parameter tuning $\Delta X / X$ worse than $1 \%$ (see the text). Light (green) dots correspond to configurations with a light Higgs $m_{h}<113 \mathrm{GeV}$. 
bounds is plotted versus $\theta_{\mu}$. Points which do not survive a $1 \%$ change are not plotted in the last

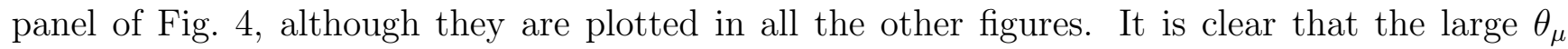
solutions require more tuning that the low $\theta_{\mu}$ solutions. Overall, we find that $\{27 \%, 46 \%, 74 \%\}$ of the (large plus small $\theta_{\mu}$ ) solutions require worse than $\{1 \%, 2 \%, 5 \%\}$ tuning (corresponding to the unplotted points, points at 0.01 , points at 0.02$\}$ in the last panel of Fig. 田. The tuning level of the solutions can also be seen in the other panels of Figs. 4 , where the open circles have tuning worse than at the level of $1 \%$.

We emphasize the importance of having a third independent constraint on the phases, from the ${ }^{199} \mathrm{Hg}$ EDM. Fig. 7 shows the result of a smaller scan of the 15-parameter space in which we only impose the limits from the electron and neutron EDMs and plot $\theta_{\mu}$ vs. $\tan \beta$ for the resulting set. The light (green) points are those which would be forbidden by the further imposition of the ${ }^{199} \mathrm{Hg}$ EDM constraints, while the dark (black) points are those which satisfy the ${ }^{199} \mathrm{Hg}$ EDM constraints. The open circles yield $m_{h}<113 \mathrm{GeV}$. We find that of the 174,000 points satisfying the neutron and electron EDM constraints (of which only 50,000 are plotted), only 4700, or 2.7\%, additionally satisfy the ${ }^{199} \mathrm{Hg}$ EDM constraint. The remaining points at large $\theta_{\mu}$ typically have a very heavy squark $(>800 \mathrm{GeV})$ or two heavy charginos $(>500 \mathrm{GeV})$. Clearly, ignoring [30 the ${ }^{199} \mathrm{Hg}$ EDM constraints allows many configurations that are experimentally forbidden, particularly for low to moderate values of the masses, and for larger values of $\tan \beta$ combined with large phases, where the regions satisfying the electron and neutron EDM constraints are small. Given the significant effect of the mercury constraint, an improved calculation of the strength of the $T$-odd nuclear
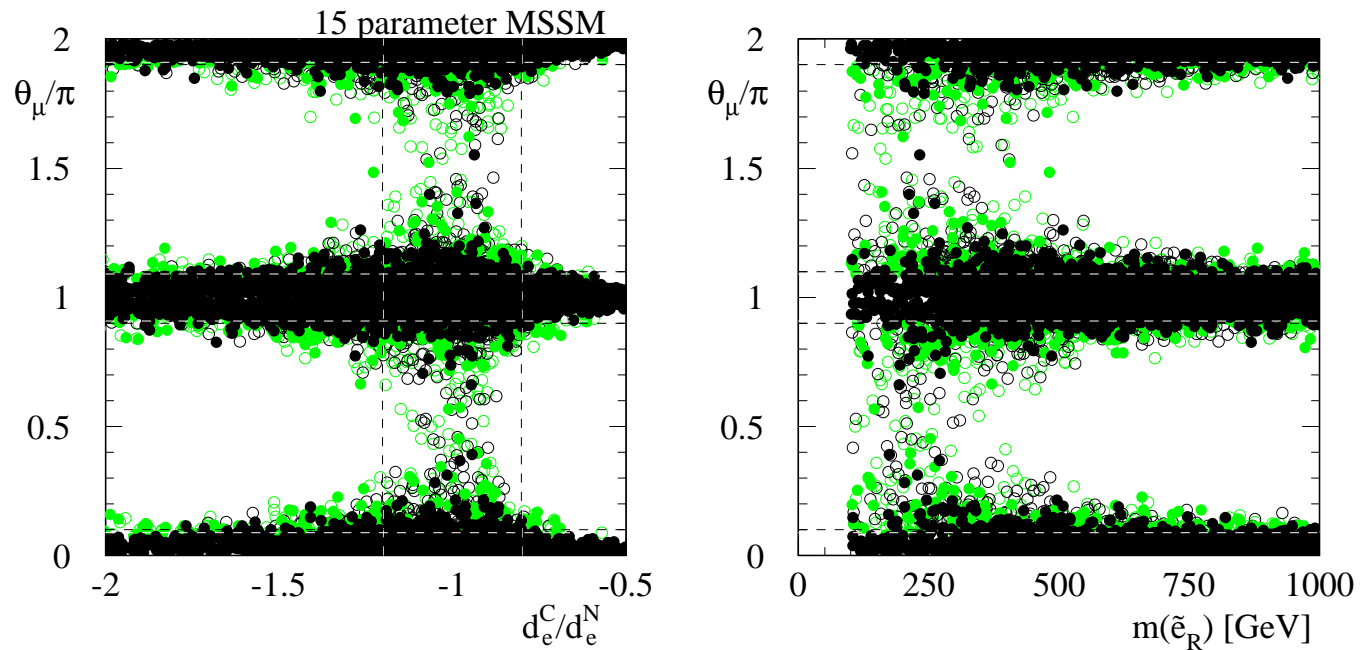

Figure 6: For the 15-parameter MSSM scan, the effect of cancellations on the allowed range of $\theta_{\mu}$. Open circles suffer from parameter tuning $\Delta X / X$ worse than $1 \%$ (see the text). Light (green) dots correspond to configurations with a light Higgs $m_{h}<113 \mathrm{GeV}$. 
forces [31] and Schiff moment of the mercury nucleus [32] will likewise be important.

We also emphasize the significance of the recent improved calculation of the neutron EDM [20, 21]. In Fig. 8 we display the values of the neutron EDM as computed using QCD sum rules in [20, 21] against those estimated using naïve dimensional analysis (NDA), as in [2]. Here we have plotted just those sets for which the neutron EDM constraint is satisfied according to either one or both of the calculational methods. We see that NDA typically overestimates the neutron EDM by roughly a factor of two, although for solutions near regions of cancellations, the discrepancy between the two can be much greater.

\subsection{3-parameter MSSM}

We lastly consider a very general model with 23 free parameters. We now allow the left and right sfermion masses to vary independently, and we take independent stop masses in computing the neutron EDM. We also take the phase and magnitude of all the $A_{i}, i=e, d, u, t$ as independent, giving a total of 7 phases, 15 mass parameters and $\tan \beta$ as the free parameters of the model. We perform a Monte Carlo scan of roughly 600 million parameter sets, over the following ranges:
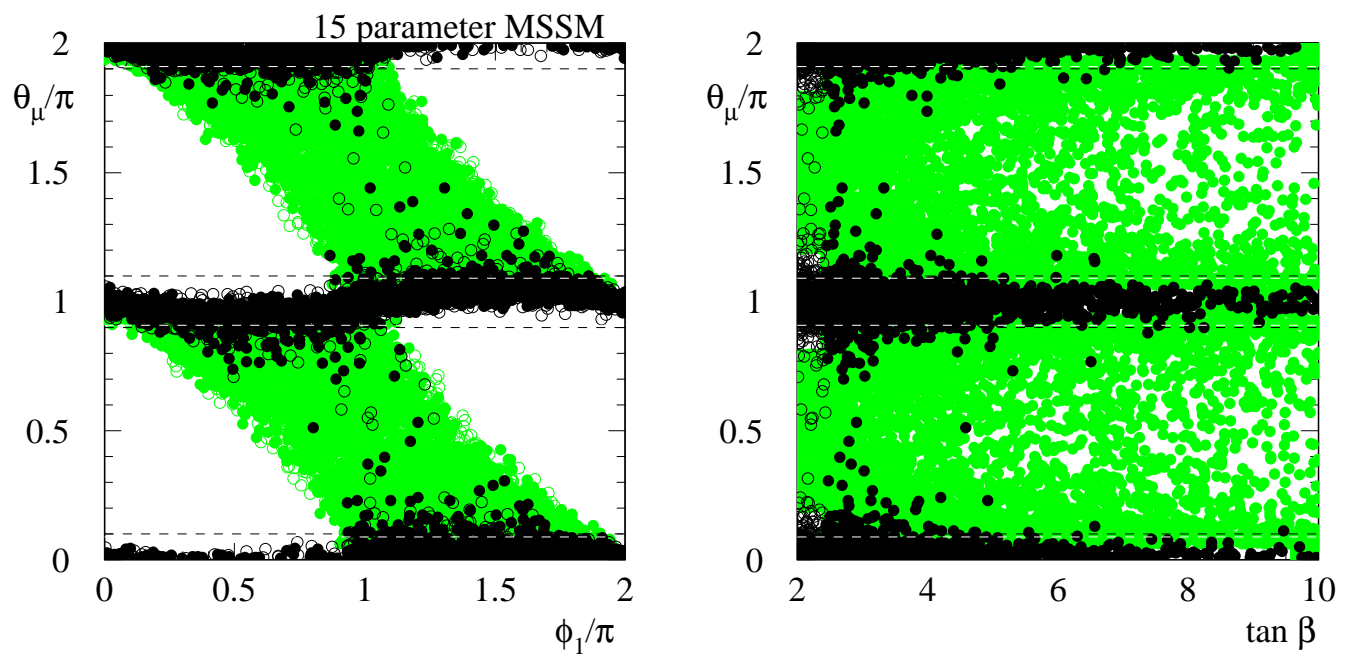

Figure 7: A scan of the 15-parameter MSSM imposing only the electron and neutron EDM constraints. Light (green) points are forbidden by the ${ }^{199} \mathrm{Hg}$ EDM constraints, while the dark (black) points satisfy the ${ }^{199} \mathrm{Hg}$ EDM constraints. The open circles have $m_{h}<113 \mathrm{GeV}$. 


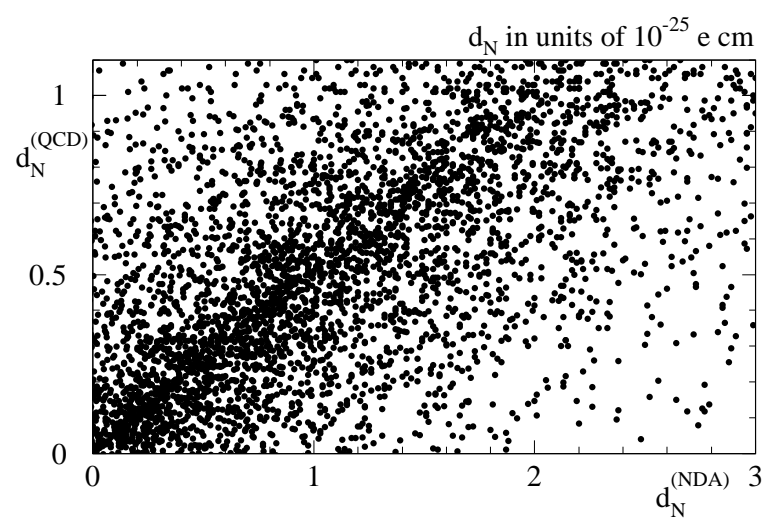

Figure 8: Computed values for the neutron EDM, using QCD sum rules (y-axis) and naïve dimensional analysis (x-axis), in units of $10^{-25} e \cdot \mathrm{cm}$.

\begin{tabular}{|c|c|c|c|c|}
\hline 0 & $\leq$ & $\theta_{\mu}, \theta_{M_{i}}, \theta_{A_{i}}$ & $\leq$ & $2 \pi$ \\
\hline $100 \mathrm{GeV}$ & $\leq$ & $\mu$ & $\leq$ & $1 \mathrm{TeV}$ \\
\hline $100 \mathrm{GeV}$ & $=$ & $2 M_{1}, M_{2}, M_{3}$ & & $1 \mathrm{TeV}$ \\
\hline $0 \mathrm{GeV}$ & $\leq$ & $\left|A_{i}\right|$ & & $\mathrm{TeV}$ \\
\hline $0 \mathrm{GeV}$ & $\leq$ & $m_{\tilde{\ell}_{L}}, m_{\tilde{e}_{R}}$ & & $1 \mathrm{TeV}$ \\
\hline $0 \mathrm{GeV}$ & $\leq$ & $m_{\tilde{q}_{L}}, m_{\tilde{u}_{R}}, m_{\tilde{d}_{R}}$ & & $1 \mathrm{TeV}$ \\
\hline $0 \mathrm{GeV}$ & & $m_{\tilde{q}_{L}^{3}}, m_{\tilde{t}_{R}}$ & & $1 \mathrm{TeV}$ \\
\hline 2 & 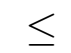 & $\tan \beta$ & & 10 \\
\hline
\end{tabular}

In Fig. 9 we display the 10,000 parameter sets satisfying the EDM constraints (1)-(3). The results are very similar to those in the case of the 15-parameter MSSM.

\section{Mass and Cross Section Measurements}

We now turn to the actual determination of the phase parameters at a future Linear Collider [33]. In an earlier work [4] it was shown that large phase values, as well as the real parameters in the neutralino/chargino sector, can be extracted to high accuracy from measured masses and cross sections, using a global fit. Their extraction from a much reduced set of observables, such as the neutralino and chargino masses alone, appears to be impossible, due to experimental uncertainties propagating from the measurement of masses and cross sections into the fitted phase parameters. Only if the uncertainty is considerably smaller than the actual phase value, do we regard the phase as being observable.

\subsection{Complete Set of Observables}

The central scenario in our analysis is a generic set of MSSM parameters derived from the unified model parameters $m_{1 / 2}=200 \mathrm{GeV}, m_{0}=100 \mathrm{GeV}, A_{0}=0, \tan \beta=4$, and $\mu>0$. The 

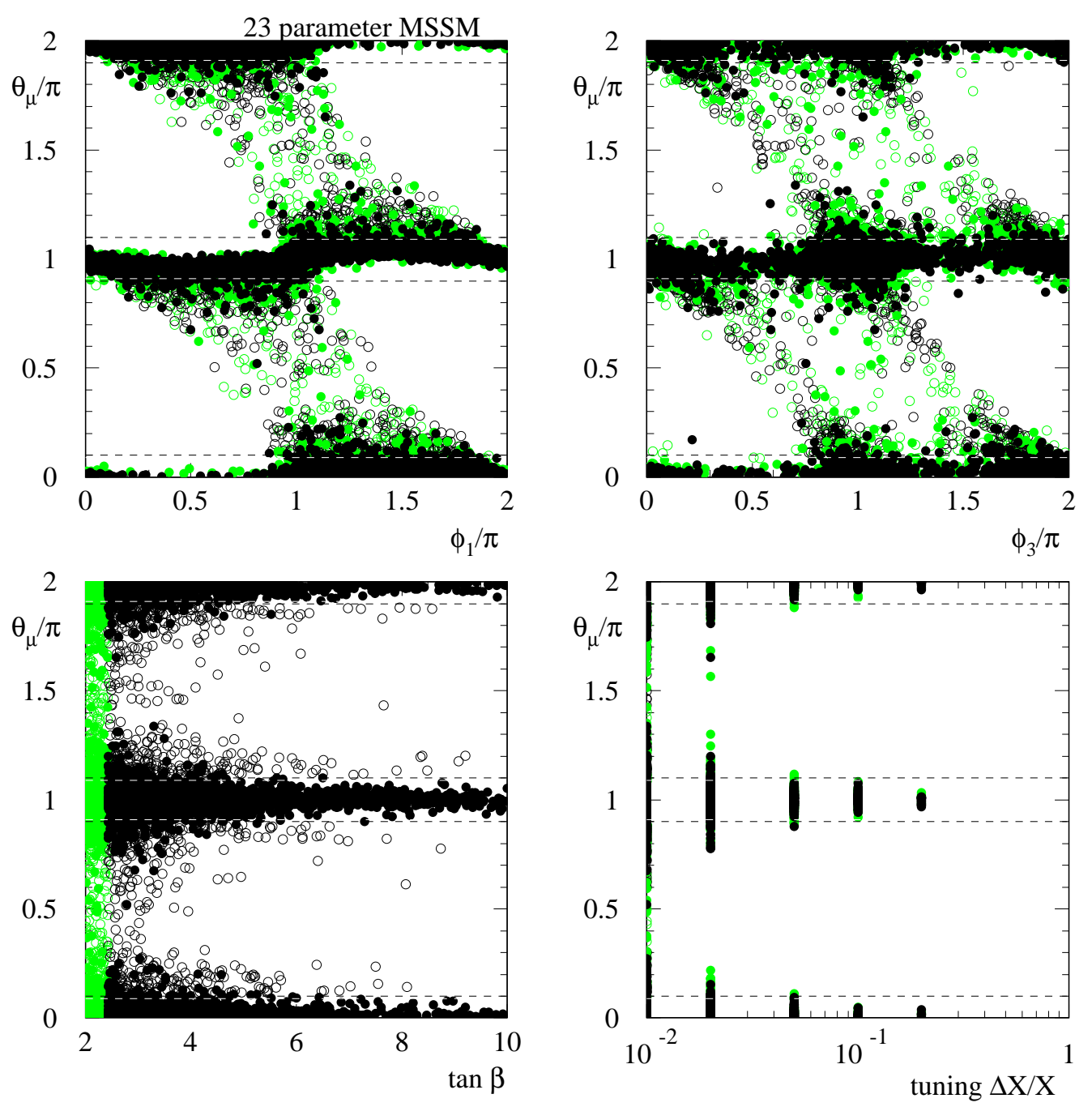

Figure 9: Parameter sets in the 23-parameter MSSM satisfying the experimental limits on the electron, neutron and ${ }^{199} \mathrm{Hg}$ EDMs. Open circles suffer from parameter tuning $\Delta X / X$ worse than 1\% (see the text). Light (green) dots correspond to configurations with a light Higgs $m_{h}<$ $113 \mathrm{GeV}$. The tuning parameter $\Delta X / X$ is defined in the text, and corresponds to the maximum variation that the point survives.. 
neutralino/chargino mass parameters are $\left|M_{1}\right|=83 \mathrm{GeV}, M_{2}=165 \mathrm{GeV}$ and $\mu=310 \mathrm{GeV}$, and the corresponding slepton masses are $m_{\tilde{e}_{L}}=180 \mathrm{GeV}, m_{\tilde{e}_{R}}=132 \mathrm{GeV}$ and $m_{\tilde{\nu}}=166 \mathrm{GeV}$. The masses of the neutralinos and charginos are given in Table 2. For the phase values $\phi_{1}=\theta_{\mu}=\pi / 10$, this scenario was investigated in our previous analysis: the error on the fitted mass parameters $\left|M_{1}\right|, M_{2},|\mu|$ is smaller than $1 \mathrm{GeV}$, and the error on the extraction of $\tan \beta$ is less than $10 \%$. The RMS of the phase determination depends on the energy and the luminosity of the collider. For a $\left(500 \mathrm{GeV}, 500 \mathrm{fb}^{-1}\right)$ machine we obtain $\phi_{1} / \pi=0.1 \pm 0.03$ and $\theta_{\mu} / \pi=0.1 \pm 0.05$, whereas for $\left(1 \mathrm{TeV}, 1000 \mathrm{fb}^{-1}\right)$, the errors are $\phi_{1} / \pi=0.1 \pm 0.05$ and $\theta_{\mu} / \pi=0.1 \pm 0.06$. The $t$-channel slepton masses are assumed to be measured in threshold scans, and the propagation of the uncertainty of their mass measurements into the phases can be neglected. We find that the statistical errors on the phase determination $\Delta \phi_{1} \sim 0.03 \pi$ and $\Delta \theta_{\mu} \sim 0.05 \pi$ are essentially independent of the central values of the phases. This implies that even with a maximal set of observables, phase values smaller than $\pi / 10$ are hidden by experimental errors and are therefore unobservable: $\mathrm{CP}$ phases of that size could, from a collider phenomenology point of view, as well be zero.

A naïve view of the inversion problem is given by the graphs in Fig. 10. We fix the parameters in the neutralino and chargino mass matrices and plot the production cross section $\sigma\left(e^{+} e^{-} \rightarrow \tilde{\chi}_{1}^{0} \tilde{\chi}_{2}^{0}\right)$ and the branching fraction $\mathrm{BR}\left(\tilde{\chi}_{2}^{0} \rightarrow \tilde{\chi}_{1}^{0} e^{+} e^{-}\right)$as a function of the phase $\phi_{1}$. The dependence on $\phi_{1}$ is strong in both observables and an analysis could be straightforward. However, the plots also show that the physical masses in the process are not constant with varying phases, either. The main problem of the analysis therefore becomes the separation of direct effects of the phase and indirect effects, where the phase changes the physical neutralino masses and these affect the cross sections and branching fractions.

\footnotetext{
${ }^{3}$ For both colliders the masses are determined in a threshold scan. For higher energy and fixed masses the cross sections decrease. Increasing the luminosity does not compensate for the increase in energy in this case.
}
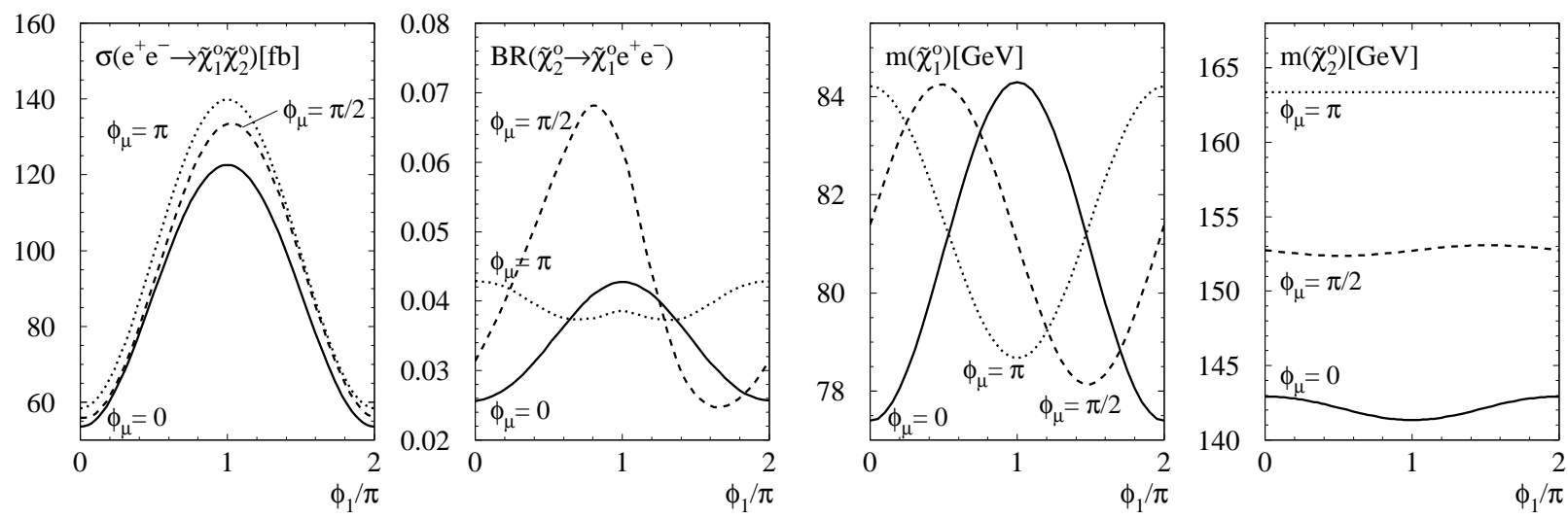

Figure 10: The masses and cross sections at a $400 \mathrm{GeV}$ Linear Collider for the scenarios discussed in the text. 
Hence, a key ingredient of the full analysis [4] as well as of the one presented in this paper is the measurement of masses through threshold scans [34]. We use the error estimates for an mSUGRA scenario with $m_{0}=100 \mathrm{GeV}, m_{1 / 2}=200 \mathrm{GeV}$ and $\tan \beta=4$. They are $0.05 / 0.07 / 0.3 / 0.6 \mathrm{GeV}$ for the neutralino masses and $0.035 / 0.25 \mathrm{GeV}$ for the chargino masses [34]. We furthermore assume the uncertainty of cross section measurements to be purely statistical. This limits the pull of small cross sections. The invisible cross section for the production of two lightest neutralinos (LSPs) is not part of our sample. For a high energy collider, the full analysis includes twelve cross section and six mass observables, determining six model parameters: $\left|M_{1}\right|, M_{2},|\mu|, \tan \beta, \phi_{1}, \theta_{\mu}$.

\subsection{Inclusion of the EDM limits}

To cover a large range of slepton masses, we consider two modifications of the central scenario: (1) the first generation sleptons are still light but just escape detection at a low energy collider. The so increased slepton masses correspond to having their $m_{0}=200 \mathrm{GeV}, m_{1 / 2}=200 \mathrm{GeV}$, i.e. $m_{\tilde{e}_{L}}=250 \mathrm{GeV}, m_{\tilde{e}_{R}}=218 \mathrm{GeV}$ and $m_{\tilde{\nu}}=240 \mathrm{GeV}$. (2) All sleptons decouple from the theory entirely, which corresponds to masses above $\mathcal{O}(1 \mathrm{TeV})$. The cross sections accessible at a $400 \mathrm{GeV}$ collider are given in Table 2 for all three sets of lepton masses. This collider energy enables us to observe one higgsino type neutralino directly, whereas the other two higgsino states are kinematically inaccessible. The cross sections are dominated by the $t$-channel process due to the gaugino nature of the light neutralino. In contrast, the production of two light charginos involves both diagrams: for light sleptons, the $t$-channel graph is large whereas in the decoupling limit the $s$-channel contribution dominates; for intermediate slepton masses destructive interference reduces the cross section by almost a factor of two. Still, the chargino cross section is by far the largest. If the lightest chargino mass is known through a threshold scan, this signature should serve to

\begin{tabular}{|r|r||r|r|r|r|}
\hline & $m_{\tilde{\chi}}[\mathrm{GeV}]$ & & $\sigma_{\text {tot }}[\mathrm{fb}], \tilde{\ell}$ light & $\sigma_{\text {tot }}[\mathrm{fb}], \tilde{\ell}$ heavy & $\sigma_{\text {tot }}[\mathrm{fb}], \tilde{\ell}$ decoupled \\
\hline \hline$\tilde{\chi}_{1}^{0}$ & 77.3 & $\left(\tilde{\chi}_{1}^{0} \tilde{\chi}_{1}^{0}\right.$ & 320.0 & 186.6 & 0.09 \\
$\tilde{\chi}_{2}^{0}$ & 142.6 & $\tilde{\chi}_{1}^{0} \tilde{\chi}_{2}^{0}$ & 87.8 & 55.3 & 0.28 \\
$\tilde{\chi}_{3}^{0}$ & 315.5 & $\tilde{\chi}_{1}^{0} \tilde{\chi}_{3}^{0}$ & 6.4 & 3.8 & 1.7 \\
$\tilde{\chi}_{4}^{0}$ & 343.0 & $\tilde{\chi}_{2}^{0} \tilde{\chi}_{2}^{0}$ & 107.8 & 64.5 & 0.16 \\
$\tilde{\chi}_{1}^{+}$ & 140.7 & $\tilde{\chi}_{1}^{+} \tilde{\chi}_{1}^{-}$ & 806.9 & 570.8 & 854.6 \\
$\tilde{\chi}_{2}^{+}$ & 431.6 & & & & \\
\hline
\end{tabular}

Table 2: Neutralino/chargino masses and cross sections at a $400 \mathrm{TeV}$ Linear Collider for the three scenarios under consideration. The production cross section of two LSPs is not part of the set of observables. 
determine the mass of the sneutrino with high precision [35].

Using the results of the EDM analysis allows us to modify the full analysis described in the previous section: we know that the phase $\theta_{\mu}$ has to be smaller than $\pi / 10$, after taking into account three conditions: (1) the experimental limits on the electron, neutron and mercury EDM have to be respected simultaneously; (2) the degree of fine tuning is limited by requiring that the solutions be stable with respect to changes of $\Delta X / X=1 \%$ in all model parameters; (3) a minimal set of final states is produced at a Linear Collider with a fixed design energy, e.g. $400 \mathrm{GeV}$. We will show that this minimal set of observables has to include a handle on one higgsino component, to allow for a determination of $\mu$. Under these assumptions, we try to determine CP-violating phases at a comparably low energy collider, which would not cover all neutralino/chargino and slepton thresholds. The reduced set of observables is supplemented by EDM constraints: $\tan \beta$ has to be small ( $\lesssim 10$ ), and may be known from the Higgs sector [36] if the Higgs bosons are sufficiently light. Since $\theta_{\mu}$ turns out to be constrained to be smaller than its minimum visible value $\pi / 10$ [4], we fix it to zero. The phase $\phi_{1}$ can be treated as independent of all other parameters in the neutralino and chargino mass matrices, since parameters correlated to $\phi_{1}$ by the EDM constraints, like $\phi_{3}$ or $\phi_{A}$, do not appear in this sector.

To determine the phases we modify our full analysis: the set of observables is first reduced to the three lightest neutralinos and the lightest chargino. Producing them in pairs yields four cross sections, i.e. eight independent observables. In the fit, we determine the real parameters $\left|M_{1}\right|, M_{2}, \mu, \phi_{1}$ as well as two $t$-channel selectron masses. For a low energy collider, the selectron masses cannot be expected to be measured in threshold scans, and this leaves us with altogether six unknown model parameters. Using $S U(2)$ symmetry the sneutrino mass can be related to the left handed selectron mass:

$$
m_{\tilde{\nu}}^{2}=m_{\tilde{e}_{L}}^{2}+m_{W}^{2} \cos ^{2} \theta_{w} \cos 2 \beta .
$$

In contrast to the complete parameter fit, the extraction is now limited by the number of fitted parameters, and the $\chi^{2}$ distribution of the best fit might be flat in certain parameters. This makes it technically difficult to add $\tan \beta$ to the set of fitted parameters, and we have to rely on a measurement in the Higgs sector. However, in principle one can extend the number of observables by non-trivial distributions, asymmetries or additional cross sections, and one can choose a fitting algorithm better suited for the problem to include $\tan \beta$ in the fit [8].

\subsection{Statistical Uncertainties}

The inclusion of experimental errors follows the same path as in the previous analysis [4]: we assume Gaussian errors for the measured masses and cross sections and define smeared pseudomeasurements. First we define a set of 'true' model parameters $\left|M_{1}\right|, M_{2} \ldots$ They predict a set 
of 'true' observables (masses and cross sections), all of which are assumed to have a Gaussian error distribution with a known width [34]. Using these distributions, we randomly vary 10000 pseudo-measurements of the set of observables. These sets become slightly inconsistent, but using a global fit we can extract the central value of every model parameter and obtain a distribution for each of themf. In the fit we minimize $\chi^{2}=\sum_{i}\left(x_{\text {reconstr, } \mathrm{i}}-x_{\text {meas }, \mathrm{i}}\right)^{2} / e_{\mathrm{i}}^{2}$, where $x_{\text {reconstr }}$ are the reconstructed observables and $x_{\text {meas }}$ are the smeared pseudo-measurements. The error on the cross sections is given as a function of the luminosity by $e_{\mathrm{i}}=\sigma_{\mathrm{i}} /(\epsilon \mathcal{L})$, where the efficiency is assumed to be $\epsilon=10 \%$. If the central value of the distribution for a given parameter agrees with its 'true' value, the statistical treatment is justified, and the width of the distribution describes the migration of observational errors into the model parameters. The final curve does not necessarily have to be Gaussian, since correlations, together with the range of the fit, can alter the shape of the curve. Hence, we quote the RMS value instead of the standard deviation of a fitted Gaussian distribution. We note that for some scenarios there exist several $\chi^{2}$ minima in different parameter regions. This is a technical complication, and the minima should be distinguishable through the actual values of $\chi^{2}$. In these cases, we limit our range of fitted parameters, making sure that the range is much bigger than the distribution we finally obtain.

The distribution of best fits to a set of 10000 pseudo-measurements is given in Fig. 11. In contrast to the earlier analysis we assume $\theta_{\mu}=0$ and rely on a known value of $\tan \beta$. The sleptons are kinematically inaccessible, and their masses have to be indirectly determined from the cross section measurement. Comparing the errors on the three mass parameters $\left|M_{1}\right|, M_{2}, \mu$ we notice the striking accuracy of the measurement of $M_{2}$. This reflects the small number of unknown parameters in the chargino mass matrix and in the $\tilde{\chi}_{1}^{+} \tilde{\chi}_{1}^{-}$production cross section: the phase $\theta_{\mu}$ and $\tan \beta$ are both fixed, and only $M_{2}$ and $\mu$ and the sneutrino mass have to be determined from the chargino mass and cross section. However, if one is able to determine the cross sections for polarized electrons and positrons and separate the $s$ and $t$-channel contributions, this accuracy will improve even further. On the other hand, if one is forced to determine $\tan \beta$ from the neutralino/chargino sector, the error on $M_{2}$ will be $\mathcal{O}(1 \mathrm{GeV})$ again. In contrast to the full analysis, the fitted values of $\left|M_{1}\right|$ and $\phi_{1}$ are now correlated. Correlations like that are a general feature of a smaller set of observables and a smaller dimension of the fit. The error on the determination of the slepton masses is not symmetric: the left selectron mass is tied to the sneutrino mass and therefore extracted from the well determined chargino sector. The right selectron mass, in contrast, has to be determined from the neutral gaugino cross sections, which are smaller by almost one order of magnitude.

The distribution of best fits for $\phi_{1}$ exhibits a similar shape to the result of the full analysis. For

\footnotetext{
${ }^{4}$ Extracting these values from the low energy parameters without the smearing would merely serve as a check of the fitting program.
} 
large luminosity of $500 \mathrm{fb}^{-1}$ it approaches a Gaussian with a RMS of 0.032 and a fitted standard deviation of 0.028. At smaller luminosity, this distribution broadens, e.g. to a RMS value of 0.037 for $300 \mathrm{fb}^{-1}$, as given in Table 3. From the cross sections in Table 2 one can see that there is a minimal luminosity at which the number of events in $\left(\tilde{\chi}_{1}^{0} \tilde{\chi}_{3}^{0}\right)$ becomes marginal. In this case there will be hardly any information on $\mu$ left in the sample. The same problem arises once the energy is too small to produce a state with significant higgsino content. If the higgsinos are almost entirely decoupled, the uncertainty on the real mass parameters becomes too large to extract the phases. Technically, the correct minimum in $\chi^{2}$ will not be found then by the fitting procedure. In Figure 10 we see that for many observables the point $\phi_{1}=0$ is extremal, i.e. a considerable number of best fits will give this result. This occurs for example in the case of low luminosity, Fig. 11. On the other hand we notice that in the set of observables under consideration, the gaugino sector is still over-determined: except for the less important slepton masses the $\tilde{\chi}_{2}^{0} \tilde{\chi}_{2}^{0}$ production cross section carries similar information as the much larger $\tilde{\chi}_{1}^{+} \tilde{\chi}_{1}^{-}$cross section. Removing the $\tilde{\chi}_{2}^{0} \tilde{\chi}_{2}^{0}$ cross section from the sample increases the RMS value given in Table 3 to 0.036 ; however, in most models $\tilde{\chi}_{2}^{0} \tilde{\chi}_{2}^{0}$ will be visible at any collider that produces pairs of light charginos.
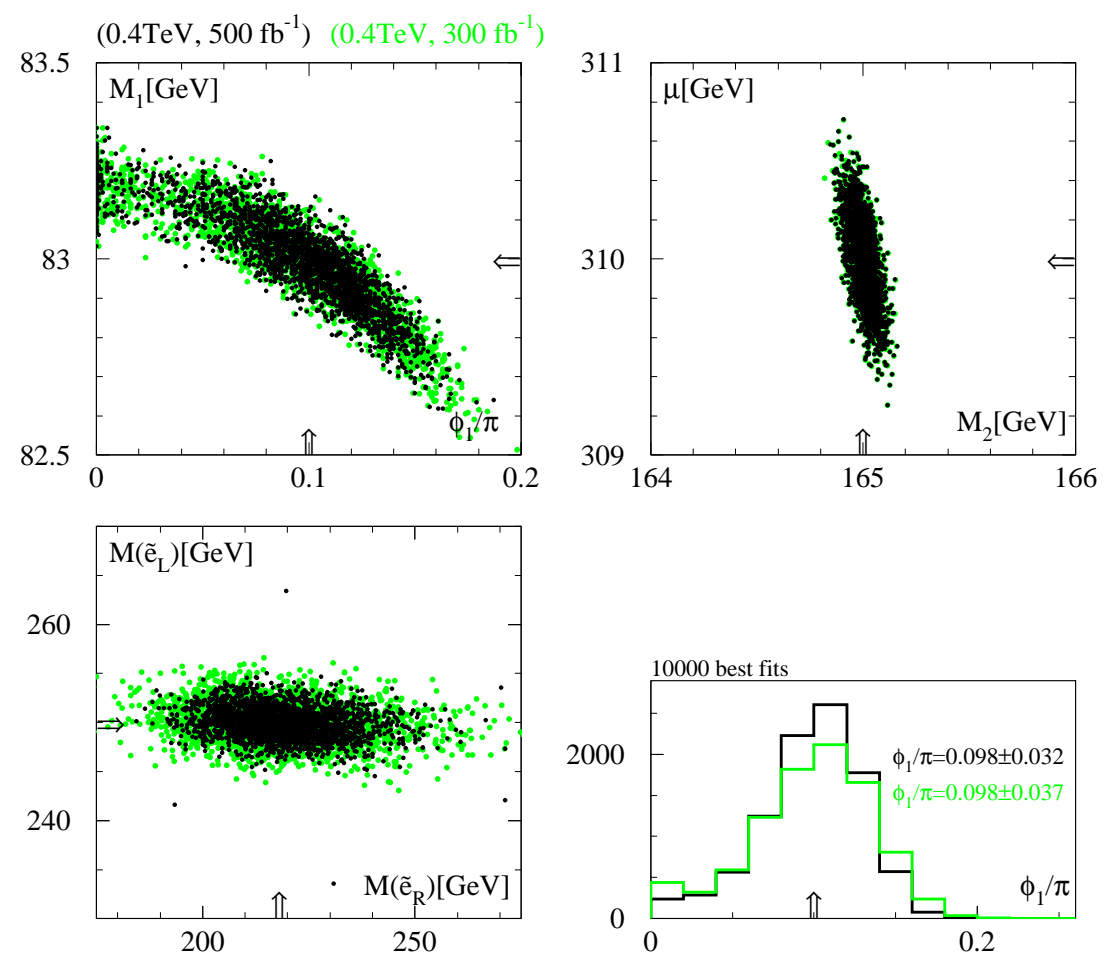

Figure 11: Parameters obtained from best fits to 10000 pseudo-measurements. The central scenario is indicated by arrows on the axes. Only one Higgsino $\tilde{\chi}_{3}^{0}$ is assumed to be observed. The black and grey (green) points correspond to two different collider designs. 


\subsection{Systematic Uncertainties}

As mentioned above the errors derived above do not take into account any systematical errors from using a wrong value e.g. of $\tan \beta$. Neither do they include an estimate what would happen if $\theta_{\mu}$ were actually small but non-zero. Both effects would lead to systematic errors and thereby to wrong central values of the 10000 fits. In Table 3 we show that fitting a set of parameters with a 'true' value $\theta_{\mu}=\pi / 20$, but assuming $\theta_{\mu}=0$ hardly affects the fit. This observation serves as a consistency check of the ansatz, namely that a small $\theta_{\mu}$ has no effect on the extraction of $\phi_{1}$ and certainly not on the dominating real parameters in the mass matrices. Analyzing the set of observables with a wrongly assumed values of $\tan \beta=3.8$ or $\tan \beta=4.2$ instead of a 'true' value of 4.0 leads to the systematic error shown in Fig 12: the spread of the best fits in the mass parameters as well as in the phase is very similar to the case where both parameters, $\theta_{\mu}$ and $\tan \beta$ are input correctly, but the central values are shifted. The central values for wrongly assumed values $\tan \beta=3.8$ and 4.2 become $\phi_{1} / \pi=0.125 \pm 0.033$ and $\phi_{1} / \pi=0.082 \pm 0.029$, i.e. they are shifted by half the RMS value.

To estimate the dependence of the analysis on the details of the underlying model, we decouple the sleptons from all cross sections. From the cross sections in Table 2, we see that the real mass
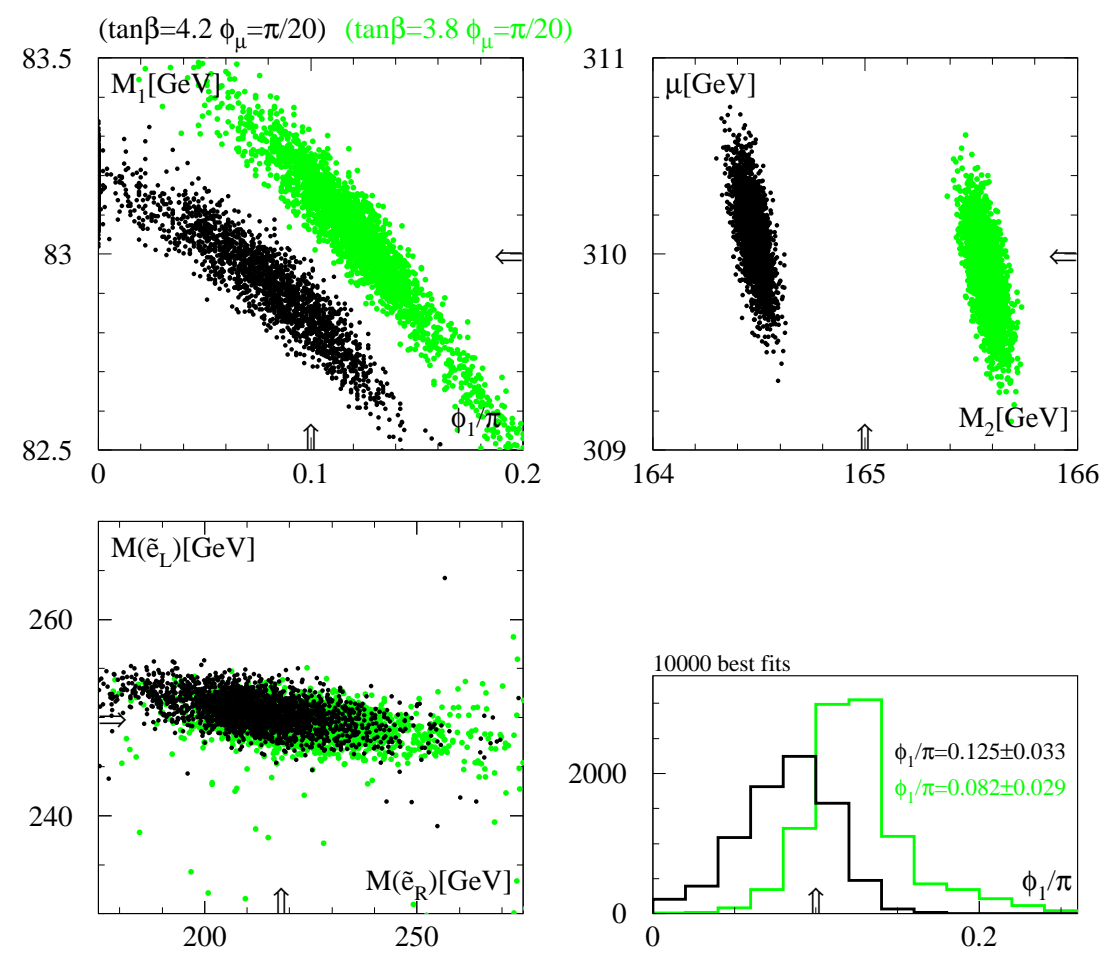

Figure 12: The effect of wrongly assumed values for $\tan \beta$ on the extraction of the model parameters. The 'true' value for $\tan \beta$ is 4 . 
parameters dominated by the physical chargino/neutralino masses and the chargino cross section should be extracted as precisely as in the case of light sleptons. However, the determination of the phase $\phi_{1}$ relies on the measurement of the $\tilde{\chi}_{1}^{0} \tilde{\chi}_{2}^{0}$ and the $\tilde{\chi}_{1}^{0} \tilde{\chi}_{3}^{0}$ cross section, where the $Z$ boson in the $s$-channel production process for neutralinos only couples to the higgsino fraction of the physical states. Since in our central scenario the light neutralinos are mainly gauginos, both of these cross sections decrease with increasing slepton masses. This leads to a RMS value of 0.044 of the $\phi_{1}$ distribution, as seen in Table 3 .

An important feature of the complete analysis [4] is that the error on the $\phi_{1}$ measurement is independent of the actual value of $\phi_{1}$. This allows us to derive lower limits on the size of observable phases, which are $\phi_{1} \gtrsim \pi / 10$ and $\theta_{\mu} \gtrsim \pi / 10$ for the complete analysis. As presented in Table 3, we obtain $\mathrm{RMS}=0.030$ for a central value of $\phi_{1}=\pi / 2$. In Figure 10, the cross section, as well as the branching fraction, tends to vary strongly around phase values of $\pi / 2$, while they become flat and extremal for positive or negative real values of $M_{1}$. This is reflected in the slightly smaller RMS value for $\phi_{1}=\pi / 2$ as compared to $\phi_{1}=\pi / 10$.

From the cross sections in Table 2 it is obvious that the determination of $\phi_{1}$ from the given set of cross sections improves, once $\mu$ takes a value closer to the gaugino mass parameters. Reducing it by a factor of two to $\mu=150 \mathrm{GeV}$ leads to a much lighter higgsino with $m_{\tilde{\chi}_{3}^{0}}=158 \mathrm{GeV}$ and an increased cross section for $\tilde{\chi}_{1}^{0} \tilde{\chi}_{3}^{0}$ production. The changes in the masses of the gaugino dominated light neutralinos are small, but the error on the $\phi_{1}$ measurement improves to 0.014.

Although it is hard to accommodate with the EDM constraints, we investigate a scenario with large $\tan \beta=30$ : from the full analysis we expect the determination of $\tan \beta$ to be less precise than for smaller values. Since all production cross sections are smaller by up to $50 \%$, the extraction of the slepton masses becomes increasingly difficult. From the analysis for small values of $\tan \beta$, however, we know that the error on the determination if the right selectron mass is larger than the mass difference between the two selectrons. One way of improving the fit for large $\tan \beta$ is therefore to assume that the selectrons are mass degenerate. With this assumption we are able to determine the phase $\phi_{1}=0.11 \pm 0.03$.

\subsection{Minimal Set of Observables}

Since the EDM constraints on $\theta_{\mu} \lesssim \pi / 10$ require the chargino sector to be essentially CPconserving, we can define a minimal set of observables sufficient to extract the phases: in the CP-conserving case one could be able to extract $|\mu|$ sufficiently precisely from a set of the mass, cross section and asymmetries in $\tilde{\chi}_{1}^{-} \tilde{\chi}_{1}^{+}$production analytically [8, 37. We use this result to investigate the phases in the neutral gaugino sector, including the masses and production cross sections of $\tilde{\chi}_{1}^{0}$ and $\tilde{\chi}_{2}^{0}$. In our central scenario (Table 2) this requires a minimum collider energy 
of $320 \mathrm{GeV}$. To the set of three cross sections and three masses we fit the neutralino parameters $\left|M_{1}\right|, M_{2}, \phi_{1}$, for given $|\mu|$, and obtain an error on the determination of $\phi_{1}$ of 0.027 , i.e. similar to case including one higgsino. Adding $\tan \beta$ to the fitted parameters increases the error on $\phi_{1}$ to $\mathrm{RMS}=0.032$, as can be seen in Fig. 13. As shown before, the inclusion of slepton masses would not change this result significantly. It only requires a larger set of observables. A wrong measurement of $|\mu|$, however, would lead to a systematic error of the phase determination. We estimate this by assuming a wrong extraction of $|\mu|=305 \mathrm{GeV}$ and $315 \mathrm{GeV}$ for a 'true' value $|\mu|=310 \mathrm{GeV}$. In Fig. 13 the extracted values of $\left|M_{1}\right|$ and $M_{2}$ are shifted systematically, as is the central value of the 10000 pseudo-measurements of $\phi_{1}$. But the shift for a $5 \mathrm{GeV}$ mismeasurement of $|\mu|$ is only by half the statistical error of the phase measurement. We therefore conclude that it is possible to extract the phase $\phi_{1}$ from a minimal set of parameters, if the error on the determination of $|\mu|$ does not exceed a few percent [37].
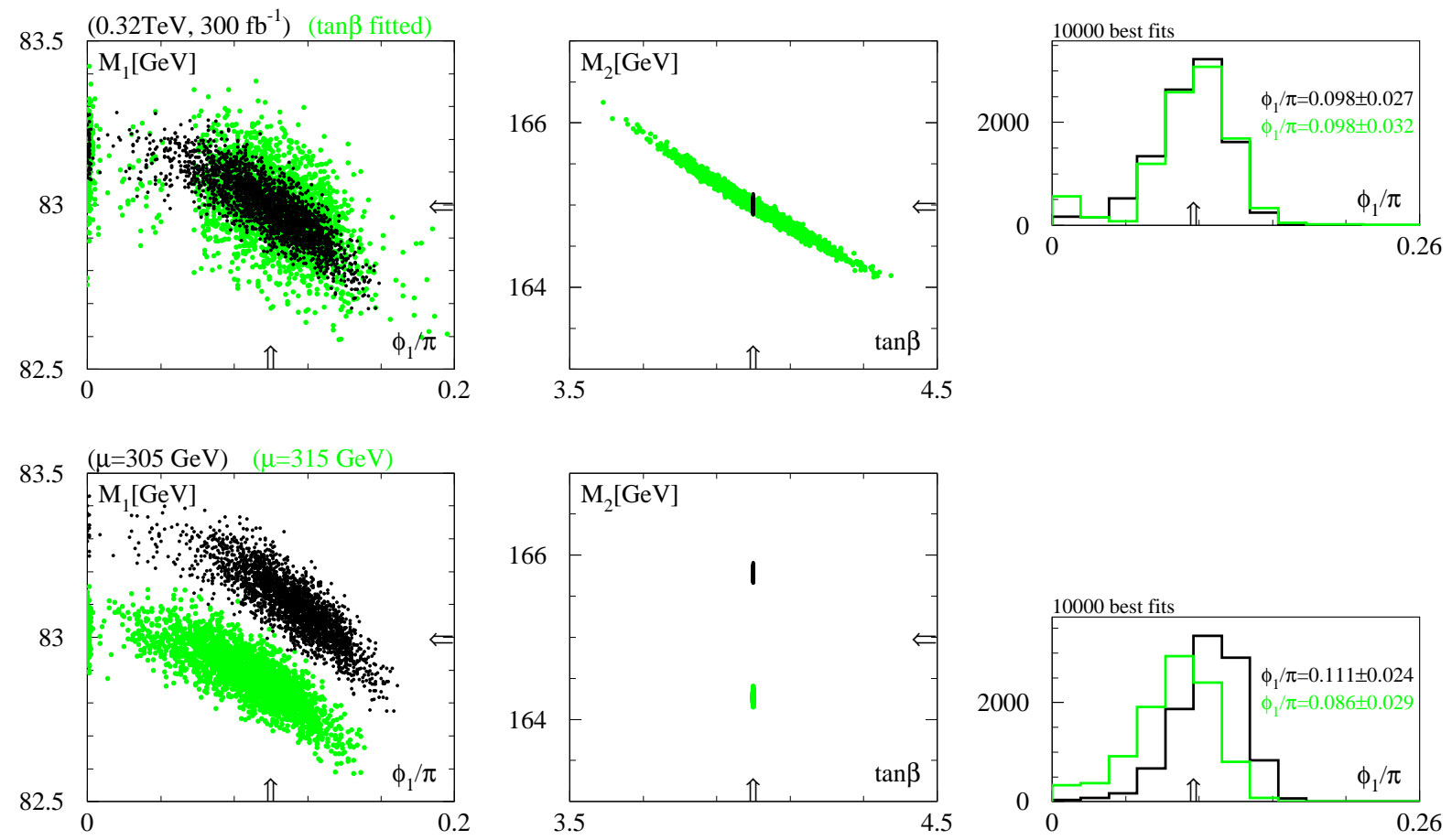

Figure 13: Top: Fit results for a known value of $\mu=310 \mathrm{GeV}$. The grey (green) points correspond to $\tan \beta$ being added to the fitted parameters. Bottom: Systematic effect from a wrong input of $|\mu|$ for known $\tan \beta$. 


\begin{tabular}{|c|c|c|c|}
\hline & & central $\phi_{1} / \pi$ & RMS \\
\hline$(1)$ & complete analysis $\left(500 \mathrm{GeV}, 500 \mathrm{fb}^{-1}\right)$ [4] & 0.097 & 0.030 \\
\hline$(2)$ & one phase $\phi_{1}$, free sleptons, fixed $\tan \beta\left(500 \mathrm{GeV}, 500 \mathrm{fb}^{-1}\right)$ & 0.099 & 0.034 \\
\hline$(3)$ & as $(2)$, but only one higgsino $\tilde{\chi}_{3}^{0}\left(400 \mathrm{GeV}, 500 \mathrm{fb}^{-1}\right)$ & 0.098 & 0.032 \\
\hline & as $(3)$, but $\left(300 \mathrm{fb}^{-1}\right)$ & 0.098 & 0.037 \\
\hline & as $(3)$, but without $\tilde{\chi}_{2}^{0} \tilde{\chi}_{2}^{0}$ production & 0.098 & 0.036 \\
\hline & as $(3)$, but 'true' $\theta_{\mu}=\pi / 20$ & 0.100 & 0.032 \\
\hline & as (3), but 'true' $\theta_{\mu}=\pi / 20$ and wrongly assumed $\tan \beta=3.8$ & 0.125 & 0.033 \\
\hline & as (3), but 'true' $\theta_{\mu}=\pi / 20$ and wrongly assumed $\tan \beta=4.2$ & 0.082 & 0.029 \\
\hline & as (3), but sleptons decoupled & 0.104 & 0.044 \\
\hline & as (3), but large phase value $\phi_{1}=\pi / 2$ & 0.501 & 0.030 \\
\hline & as $(3)$, but light higgsinos $\mu=150 \mathrm{GeV}\left(300 \mathrm{GeV}, 500 \mathrm{fb}^{-1}\right)$ & 0.100 & 0.014 \\
\hline$(4)$ & only gauginos $\left(320 \mathrm{GeV}, 300 \mathrm{fb}^{-1}\right)$ & 0.098 & 0.027 \\
\hline & as $(4)$, but $500 \mathrm{fb}^{-1}$ & 0.099 & 0.021 \\
\hline & as (4), but $\tan \beta$ fitted & 0.098 & 0.032 \\
\hline & as (4), but wrongly assumed $\mu=305 \mathrm{GeV}$ & 0.111 & 0.024 \\
\hline & as (4), but wrongly assumed $\mu=315 \mathrm{GeV}$ & 0.086 & 0.029 \\
\hline
\end{tabular}

Table 3: Central and RMS values for the distribution of $\phi_{1}$ values, fitted from the 10000 pseudoexperiments, with a "true" value of $\phi_{1}=0.1 \pi$ (except where indicated).

\section{CP-Odd Variables and Asymmetries}

To unambiguously identify an effect of CP violation, one needs to construct a "CP-odd variable", whose expectation value vanishes if $\mathrm{CP}$ is conserved. At an $e^{+} e^{-}$linear collider, the initial state can be made a $\mathrm{CP}$ eigenstate, given the $\mathrm{CP}$ transformation relation

$$
e^{-}\left(\sigma_{1}, \vec{p}\right) e^{+}\left(\sigma_{2},-\vec{p}\right) \Rightarrow e^{-}\left(-\sigma_{2}, \vec{p}\right) e^{+}\left(-\sigma_{1},-\vec{p}\right)
$$

where $\sigma_{i}$ is the fermion helicity. We consider a specific process

$$
e^{-}\left(p_{1}\right) e^{+}\left(p_{2}\right) \rightarrow \tilde{\chi}_{1}^{0} \tilde{\chi}_{2}^{0} \rightarrow \ell_{1}^{-}\left(q_{1}\right) \ell_{2}^{+}\left(q_{2}\right) \tilde{\chi}_{1}^{0} \tilde{\chi}_{1}^{0}
$$

Denote a helicity matrix element by $\mathcal{M}_{\sigma_{1} \sigma_{2}}\left(\vec{q}_{1}, \vec{q}_{2}\right)$ where $\sigma_{1}\left(\sigma_{2}\right)$ is the helicity of the initial state electron (positron), which coincides with the longitudinal beam polarization; $\vec{q}_{1}\left(\vec{q}_{2}\right)$ denotes the momentum of the final state fermion (anti-fermion). For the process of Eq. (9), only two combinations of the helicity amplitude $\mathcal{M}_{-+}, \mathcal{M}_{+-}$contribute. It is easy to show that under $\mathrm{CP}$ transformation,

$$
\mathcal{M}_{-+}\left(\vec{q}_{1}, \vec{q}_{2}\right) \Rightarrow \mathcal{M}_{-+}\left(-\vec{q}_{2},-\vec{q}_{1}\right)
$$


and $\mathcal{M}_{+-}$transforms similarly. If $\mathrm{CP}$ is conserved in the reaction, relation (10) becomes an equality. This argument is applicable for unpolarized or transversely polarized beams as well.

One can construct CP-odd kinematical variables to test the $\mathrm{CP}$ property of the reaction. We consider the following three angles defined as

$$
\cos \theta_{+}=\frac{\vec{p}_{1} \cdot \vec{q}_{+}}{\left|\vec{p}_{1}\right|\left|\vec{q}_{+}\right|}, \quad \cos \theta_{-}=\frac{\left(\vec{p}_{1} \times \vec{q}_{-}\right) \cdot\left(\vec{q}_{1} \times \vec{q}_{2}\right)}{\left|\vec{p}_{1} \times \vec{q}_{-}\right|\left|\vec{q}_{1} \times \vec{q}_{2}\right|}, \quad \cos \theta_{\ell}=\frac{\vec{p}_{1} \cdot\left(\vec{q}_{1} \times \vec{q}_{2}\right)}{\left|\vec{p}_{1}\right|\left|\vec{q}_{1} \times \vec{q}_{2}\right|},
$$

where $\vec{q}_{+}=\vec{q}_{1}+\vec{q}_{2}$ and $\vec{q}_{-}=\vec{q}_{1}-\vec{q}_{2}$. It is easy to verify that all the three variables are CP-odd under final state CP transformations. We can then construct "forward-backward" asymmetries

$$
\mathcal{A}^{F B}=\sigma^{F}-\sigma^{B}=\int_{0}^{1} \frac{d \sigma}{d \cos \theta} d \cos \theta-\int_{-1}^{0} \frac{d \sigma}{d \cos \theta} d \cos \theta
$$

with respect to a $\mathrm{CP}$-odd angle $\theta$.

Of the four Feynman diagrams contributing to process (9), there are two diagrams that contain explicit CP-violating phases, one from $s$-channel $Z$ exchange and one from a $t$-channel selectron exchange. If $\mu>\left|M_{1}\right|, M_{2}\left(\mu<\left|M_{1}\right|, M_{2}\right)$, then in most part of the parameter space the contribution from the selectron $(Z)$ exchange diagram is dominant, thus makes the CP asymmetry from the interference small. We scanned the parameter space in $\left(\phi_{1}, \theta_{\mu}\right)$, the asymmetry obtained is typically $0.1-1.5 \%$. The beam polarizations do not improve the situation significantly. As an example, for $\left|M_{1}\right|=80 \mathrm{GeV}, M_{2}=200 \mathrm{GeV}, \mu=275 \mathrm{GeV}, \tan \beta=4.0$ and $m_{\tilde{e}_{R}}=165 \mathrm{GeV}$, while $\phi_{1}=0.90 \pi$ and $\theta_{\mu}=0.25 \pi$ the asymmetry from $\cos \theta_{\ell}$ appears to be about $1.0 \%$, with an asymmetry rate of $2.7 \mathrm{fb}$. The asymmetries for other variables in Eq. (11) are comparable. Naively, such an asymmetry at a high luminosity linear collider of $200 \mathrm{fb}^{-1}$ would lead to a $2 \sigma$ statistical effect, as estimated in [30]. However, one would have to keep the systematics of the asymmetry measurements well below a percent level, in order to establish a positive observation. We thus consider it very challenging to experimentally observe this rather small asymmetry.

\section{Conclusions/Outlook}

We have shown that the current experimental limits on the neutron, electron and ${ }^{199} \mathrm{Hg}$ electric dipole moments strongly constrain general SUSY models with several CP-violating phases, even in the presence of strong cancellations between the various SUSY contributions to the EDMs. Although it is only $\theta_{\mu}$ which is typically constrained to be small, in models in which the phase of $B$ is correlated to the other (large) phases in the model, this translates into a tight restriction on some combination of (large) phases, which may not be natural in any given model. The next year will see significant improvements in the experimental limit on the electron EDM [38], and work on improving the mercury EDM limits continues as well. Further down the road, new experimental 
techniques, such as using diatomic molecules to measure the electron EDM [39], or studies of the effects of $\mathrm{CP}$ violation on other systems, such as $(b \bar{b})$ production [40, will probe additional CP violation in the MSSM to still more sensitive levels.

These constraints on CP-violating phases are important for studies at a future Linear Collider. It was shown that one can always extract the phases $\phi_{1}$ and $\theta_{\mu}$ from a complete set of masses and cross sections for neutralino and chargino pair production [由]. The new EDM constraints essentially require $\theta_{\mu}$ to be too small to be measured at a Linear Collider. In the region where charginos and neutralinos are visible at a Linear Collider $\tan \beta$ is preferably small, i.e. rendering its measurement accessible through Higgs production [36]. Using the above constraints we extracted the finite phase $\phi_{1}$ from a reduced set of masses and cross sections and find that a minimal set of observables is limited by the presence of a non-vanishing Higgsino component in the final state particles. Since a lower energy $(400 \mathrm{GeV})$ Linear Collider might not be able to produce slepton pairs we show that the $t$-channel masses in chargino and neutralino production can be fitted, their uncertainty does not interfere with the $\phi_{1}$ phase measurement. Pair production of light charginos [8] might provide us with precise indirect information on the size of $|\mu|$ [37]. We showed that in this case that we do not need to produce any chargino directly to determine the phase $\phi_{1}$ from a truly minimal set of (gaugino) observables. For this set of observables we can even measure $\tan \beta$ and easily cross check a $\tan \beta$ measurement from the Higgs sector.

We finally studied CP-odd variables at $e^{+} e^{-}$linear colliders. We found that the CP decay asymmetry constructed from the final state kinematics is typically about $0.1-1.5 \%$. It will be challenging to experimentally observe this rather small asymmetry.

\section{Acknowledgments}

We thank the organizers of the "30 Years of Supersymmetry" Workshop in Minneapolis, where some of this work was completed. We would like thank Maxim Pospelov for many discussions, along with Norval Fortson, Mike Romalis, Jan Kalinowski and Hitoshi Murayama. TF would also like to thank the TPI in Minneapolis for its gracious hospitality this fall. This work was supported in part by DOE grant DE-FG02-95ER-40896, in part by the University of Wisconsin Research Committee with funds granted by the Wisconsin Alumni Research Foundation, and in part by DOE grant DOE-EY-76-02-3071.

\section{References}

[1] T. Falk and K. Olive, Phys. Lett. B375, 196 (1996). 
[2] T. Ibrahim and P. Nath, Phys. Lett. B418, 98 (1998); T. Ibrahim and P. Nath, Phys. Rev. D57, 478 (1998); Erratum-ibid. D58, 019901 (1998); Erratum-ibid. D60, 079903 (1999); Erratum-ibid. D60, 119901 (1999).

[3] T. Ibrahim and P. Nath, Phys. Rev. D58, 111301 (1998); Erratum-ibid. D60, 099902 (1999);

M. Brhlik, G. J. Good and G.L. Kane, Phys. Rev. D59, 115004 (1999).

[4] V. Barger, T. Han, T. Li and T. Plehn, Phys. Lett. B475, 342 (2000).

[5] S. Y. Choi, A. Djouadi, H. S. Song and P. M. Zerwas, Eur. Phys. J. C8, 669 (1999); J. L. Kneur and G. Moultaka, Phys. Rev. D61, 095003 (2000); S. Y. Choi, A. Djouadi, M. Guchait, J. Kalinowski, H. S. Song and P. M. Zerwas, Eur. Phys. J. C14, 535 (2000).

[6] S. Abel et al. [SUGRA Working Group Collaboration], hep-ph/0003154.

[7] J. Ellis, J. S. Hagelin, D. V. Nanopoulos and M. Srednicki, Phys. Lett. B127, 233 (1983); V. Barger, R. W. Robinett, W. Y. Keung and R. J. Phillips, Phys. Lett. B131, 372 (1983); S. Dawson, E. Eichten and C. Quigg, Phys. Rev. D31, 1581 (1985); W. Beenakker, M. Klasen, M. Krämer, T. Plehn, M. Spira and P. M. Zerwas, Phys. Rev. Lett. 83, 3780 (1999).

[8] T. Tsukamoto, K. Fujii, H. Murayama, M. Yamaguchi and Y. Okada, Phys. Rev. D51, 3153 (1995); J. L. Feng, M. E. Peskin, H. Murayama and X. Tata, Phys. Rev. D52, 1418 (1995); S. Y. Choi, A. Djouadi, H. Dreiner, J. Kalinowski and P. M. Zerwas, Eur. Phys. J. C7, 123 (1999); G. Moortgat-Pick, H. Fraas, A. Bartl and W. Majerotto, Eur. Phys. J. C9, 521 (1999).

[9] M. V. Romalis, W. C. Griffith, and E. N. Fortson, hep-ex/0012001; see also J.P. Jacobs et al., Phys. Rev. Lett. 71, 3782 (1993) and J.P. Jacobs et al., Phys. Rev. A52, 3521 (1995).

[10] S. Dimopoulos and D. Sutter, Nucl. Phys. B452 (1995) 496.

[11] R. D. Peccei and H. R. Quinn, Phys. Rev. Lett. 38, 1440 (1977).

[12] I. Bigi and N.G. Uraltsev, Sov. Phys. JETP 100 (1991) 198; M. Pospelov, Phys. Rev. D58, 097703 (1998).

[13] J. Ellis, S. Ferrara, and D.V. Nanopoulos, Phys. Lett. 114B, 231 (1982); W. Buchmüller and D. Wyler, Phys. Lett. 121B, 321 (1983); J. Polchinski and M. Wise, Phys. Lett. 125B, 393 (1983); F. del Aguila, M. Gavela, J. Grifols, and A. Mendez, Phys. Lett. 126B, 71 (1983); D.V. Nanopoulos and M. Srednicki, Phys. Lett. 128B, 61 (1983); M. Dugan, B. Grinstein and 
L. Hall, Nucl. Phys. B255, 413 (1985); C.-S. Huang and W. Liao, Phys. Rev. D62, 016008 (2000).

[14] Y. Kizukuri \& N. Oshimo, Phys. Rev. D45, 1806 (1992); D46, 3025 (1992).

[15] T. Falk, K. A. Olive, M. Pospelov and R. Roiban, Nucl. Phys. B560, 3 (1999).

[16] K.F. Smith et al., Phys. Lett. B234, 191 (1990); I.S. Altarev et al., Phys. Lett. B276, 242 (1992).

[17] E.D. Commins et al., Phys. Rev. A50, 2960 (1994).

[18] T. Falk and K.A. Olive, Phys. Lett. B439, 71 (1998).

[19] T. Falk, K.A. Olive and M. Srednicki, Phys.Lett. B354, 99 (1995).

[20] M. Pospelov and A. Ritz, Nucl. Phys. B573, 177 (2000); M. Pospelov and A. Ritz, Phys. Rev. Lett. 83, 2526 (1999).

[21] M. Pospelov and A. Ritz, hep-ph/0010037.

[22] S. Weinberg, Phys. Rev. Lett. 63, 2333 (1989).

[23] A. Pilaftsis, Phys.Lett. B471, 174 (1999).

[24] T. Ibrahim and P. Nath, Phys. Rev. D61, 093004 (2000); M. Brhlik, L. Everett, G. L. Kane and J. Lykken, Phys. Rev. Lett. 83, 2124 (1999).

[25] K. Inoue, A. Kakuto, H. Komatsu and S. Takeshita, Prog. Th. Phys. 68, 927 (1982); M. Drees and M. Nojiri, Phys. Rev. D47 376, (1993); W. de Boer, R. Ehret, W. Oberschulte, and D.I. Kazakov, Z. Phys. C67, 647 (1995); S.P. Martin and M.T. Vaughn, Phys. Rev. D50, 2282 (1994).

[26] R. Garisto and J. D. Wells, Phys. Rev. D55, 1611 (1997).

[27] E. Accomando, R. Arnowitt and B. Dutta, Phys. Rev. D61, 115003 (2000).

[28] Recent LEP limits on SUSY particles and Higgs bosons can be found in the November LEPC talks, which can be found at http://delphiwww.cern.ch/ offline/physics_links/lepc.html

[29] S. Heinemeyer, W. Hollik and G. Weiglein, Phys. Rev. D58, 091701 (1998); see also R.-J. Zhang, Phys.Lett. B447, 89 (1999). 
[30] S.Y. Choi, H.S. Song and W.Y. Song, Phys. Rev. D61, 075004 (2000).

[31] V.M. Khatsimovsky, I.B. Khriplovich and A.S. Yelkhovsky, Ann. Phys. 186, 1 (1988).

[32] O.P. Sushkov, V.V. Flambaum and I.B. Khriplovich, Zh. Eksp. Theor. Fiz. 87, 1521 (1984) (Sov. Phys. JETP 60, 873 (1984)).

[33] D. A. Dicus, S. Nandi, W. W. Repko and X. Tata, Phys. Rev. Lett. 51, 1030 (1983); A. Bartl, H. Fraas and W. Majerotto, Z. Phys. C30, 441 (1986); A. Bartl, H. Fraas and W. Majerotto, Nucl. Phys. B278, 1 (1986); E. Accomando et al. Phys. Rept. 299, 1 (1998); T. Li, Ph.D. thesis (2000).

[34] H. Martyn and G. A. Blair, 4th International Workshop on Linear Colliders, Sitges, 1999. hep-ph/9910416.

[35] G. Moortgat-Pick and H. Fraas, Acta Phys. Polon. B30, 1999 (1999).

[36] J.L. Feng and T. Moroi, Phys. Rev. D56, 5962 (1997); V. Barger, T. Han and J. Jiang, hep-ph/0006223.

[37] J. Kalinowski, private communication.

[38] Eugene Commins, private communication.

[39] D. DeMille et al., "Investigation of Electronic Transitions in PbO", submitted to Phys. Rev. A.

[40] D. A. Demir and M. B. Voloshin, hep-ph/0012123. 